\title{
LIFTABLE VECTOR FIELDS OVER CORANK ONE MULTIGERMS
}

\author{
T. NISHIMURA, R. OSET SINHA, M. A. S. RUAS, AND R. WIK ATIQUE
}

\begin{abstract}
In this paper, a systematic method is given to construct all liftable vector fields over an analytic multigerm $f:\left(\mathbb{K}^{n}, S\right) \rightarrow\left(\mathbb{K}^{p}, 0\right)$ of corank at most one admitting a one-parameter stable unfolding.
\end{abstract}

Mathematics Subject Classification (2010): 58K40 (primary), 57R45, 58K20 (secondary).

Key words: liftable vector field, reduced Kodaira-Spencer-Mather map, higher version of the reduced Kodaira-Spencer-Mather map, finite multiplicity, corank at most one.

\section{CONTENTS}

1. Introduction

2. Proofs of Propositions 3, 4, 5, and 6

3. Examples of Theorem 1

4. Proof of Theorem 1

5. Proof of Theorem 2

6. How to construct liftable vector fields

6.1. $\operatorname{Lift}\left(\varphi_{k}\right)$ for $\varphi_{k}\left(u_{1}, \ldots, u_{k-2}, v_{1}, \ldots, v_{k-1}, y\right)=$ $\left(u_{1}, \ldots, u_{k-2}, v_{1}, \ldots, v_{k-1}, y^{k}+\sum_{i=1}^{k-2} u_{i} y^{i}, \sum_{i=1}^{k-1} v_{i} y^{i}\right)$

6.2. $\operatorname{Lift}\left(\psi_{n}\right)$ for $\psi_{n}\left(v_{1}, \ldots, v_{n-1}, y\right)=\left(v_{1}, \ldots, v_{n-1}, y^{2}, v_{1} y, \ldots, v_{n-1} y\right)$

6.3. $\operatorname{Lift}(\phi)$ for $\phi(x, y)=\left(x, y^{2}, y^{3}+x y\right) \quad 23$

6.4. Lift $(f)$ for $f(x, y)=\left\{\left(x, y^{2}\right),\left(x^{2}, y\right)\right\} \quad 24$

6.5. Lift $(f)$ for $f(x)=\left\{\left(x^{2}, x^{3}\right),\left(x^{3}, x^{2}\right)\right\} \quad 25$

6.6. $\operatorname{Lift}(f)$ for $f(y)=\left(y^{2}, 0\right)$

6.7. $\operatorname{Lift}\left(f_{k}\right)$ for $f_{k}(y)=\left(y^{2}, y^{2 k+1}\right)(k \geq 1)$

6.8. $\quad \operatorname{Lift}\left(S_{k}^{ \pm}\right)$for $S_{k}^{ \pm}(x, y)=\left(x, y^{2}, y^{3} \pm x^{k+1} y\right)(k \geq 0)$

6.9. Lift $(f)$ for $f(x, y)=\left\{\left(x, y^{3}+x y\right),\left(x, y^{2}\right)\right\}$

7. The case $n>p$

Acknowledgements 33

References

T. Nishimura is partially supported by JSPS and CAPES under the Japan-Brazil research cooperative program.

R. Oset Sinha is partially supported by FAPESP grant no. 2013/02381-1 and DGCYT and FEDER grant no. MTM2012-33073.

M. A. S. Ruas and R. Wik Atique are partially supported by FAPESP grant no. 2014/00304-2. M. A. S. Ruas is partially supported by CNPq grant no. 303774/2008-8. 


\section{INTRODUCTION}

Let $S$ be a finite subset of $\mathbb{K}^{n}$, where $\mathbb{K}$ is the real field $\mathbb{R}$ or the complex field $\mathbb{C}$ and $n$ is a positive integer. A map-germ $\left(\mathbb{K}^{n}, S\right) \rightarrow\left(\mathbb{K}^{p}, 0\right)$ is called a multigerm, and it is called a mono-germ if $S$ consists of only one point. Let $C_{S}$ (resp., $C_{0}$ ) be the set of analytic (that is, real-analytic if $\mathbb{K}=\mathbb{R}$ or holomorphic if $\mathbb{K}=\mathbb{C}$ ) multigerms of function $\left(\mathbb{K}^{n}, S\right) \rightarrow \mathbb{K}$ (resp., germs of function $\left.\left(\mathbb{K}^{p}, 0\right) \rightarrow \mathbb{K}\right)$ ), and let $m_{S}$ (resp., $m_{0}$ ) be the subset of $C_{S}$ (resp., $C_{0}$ ) consisting of analytic functiongerms $\left(\mathbb{K}^{n}, S\right) \rightarrow(\mathbb{K}, 0)$ (resp., $\left.\left(\mathbb{K}^{p}, 0\right) \rightarrow(\mathbb{K}, 0)\right)$. It is clear that the sets $C_{S}$ and $C_{0}$ have natural $\mathbb{K}$-algebra structures induced by the $\mathbb{K}$-algebra structure of $\mathbb{K}$. For an analytic multigerm $f:\left(\mathbb{K}^{n}, S\right) \rightarrow\left(\mathbb{K}^{p}, 0\right)$, let $f^{*}: C_{0} \rightarrow C_{S}$ be the $\mathbb{K}$-algebra homomorphism defined by $f^{*}(u)=u \circ f$. Set $Q(f)=C_{S} / f^{*} m_{0} C_{S}$. A multigerm $f:\left(\mathbb{K}^{n}, S\right) \rightarrow\left(\mathbb{K}^{p}, 0\right)$ is said to have finite multiplicity if $Q(f)$ is a finite dimensional $\mathbb{K}$-vector space. It is well-known that if a multigerm $f:\left(\mathbb{K}^{n}, S\right) \rightarrow\left(\mathbb{K}^{p}, 0\right)$ has finite multiplicity, then $n$ must be less than or equal to $p$.

For an analytic multigerm $f:\left(\mathbb{K}^{n}, S\right) \rightarrow\left(\mathbb{K}^{p}, T\right)$ such that $f(S) \subset T$, where $S$ (resp., $T$ ) is a finite subset of $\mathbb{K}^{n}$ (resp., $\mathbb{K}^{p}$ ), let $\theta_{S}(f)$ be the $C_{S}$-module consisting of germs of analytic vector fields along $f$. We may identify $\theta_{S}(f)$ with $\underbrace{C_{S} \oplus \cdots \oplus C_{S}}_{p \text { tuples }}$. We set $\theta_{S}(n)=\theta_{S}\left(i d \cdot\left(\mathbb{K}^{n}, S\right)\right)$ and $\theta_{0}(p)=\theta_{\{0\}}\left(i d \cdot\left(\mathbb{K}^{p}, 0\right)\right)$, where $i d \cdot\left(\mathbb{K}^{n}, S\right)$ (resp., $i d \cdot\left(\mathbb{K}^{p}, 0\right)$ ) is the germ of the identity mapping of $\left(\mathbb{K}^{n}, S\right)$ (resp., $\left.\left(\mathbb{K}^{p}, 0\right)\right)$.

For a given analytic multigerm $f:\left(\mathbb{K}^{n}, S\right) \rightarrow\left(\mathbb{K}^{p}, 0\right)$, following Mather $([14)$, we define $t f$ and $\omega f$ as follows:

$$
\begin{array}{ll}
t f: \theta_{S}(n) \rightarrow \theta_{S}(f), & t f(\eta)=d f \circ \eta, \\
\omega f: \theta_{0}(p) \rightarrow \theta_{S}(f), & \omega f(\xi)=\xi \circ f,
\end{array}
$$

where $d f$ is the differential of $f$. For $f$, following Wall $([28])$, we set

$$
\begin{aligned}
T \mathcal{R}(f)=t f\left(m_{S} \theta_{S}(n)\right), & T \mathcal{R}_{e}(f)=t f\left(\theta_{S}(n)\right), \\
T \mathcal{L}(f)=\omega f\left(m_{0} \theta_{0}(p)\right), & T \mathcal{L}_{e}(f)=\omega f\left(\theta_{0}(p)\right), \\
T \mathcal{A}(f)=T \mathcal{R}(f)+T \mathcal{L}(f), & T \mathcal{A}_{e}(f)=T \mathcal{R}_{e}(f)+T \mathcal{L}_{e}(f), \\
T \mathcal{K}(f)=T \mathcal{R}(f)+f^{*} m_{0} \theta_{S}(f), & T \mathcal{K}_{e}(f)=T \mathcal{R}_{e}(f)+f^{*} m_{0} \theta_{S}(f) .
\end{aligned}
$$

For a given analytic multigerm $f:\left(\mathbb{K}^{n}, S\right) \rightarrow\left(\mathbb{K}^{p}, 0\right)$, following Arnol'd ([1]), we call a vector field $\xi \in \theta_{0}(p)$ liftable over $f$ if $\xi \circ f$ belongs to $T \mathcal{R}_{e}(f)$. The set of vector fields liftable over $f$ is denoted by $\operatorname{Lift}(f)$. It is clear that $\operatorname{Lift}(f)$ naturally has a $C_{0}$-module structure.

The use of liftable vector fields has proven to be a fundamental tool in the study of classification techniques. In [1] and [6], and more recently in [5, [10] and [22, $\operatorname{Lift}(f)$ has played a central role in the development of operations and in order to calculate the codimensions of the multigerms resulting from these operations. However, in general, obtaining generators of $\operatorname{Lift}(f)$ can be a very hard task. In fact, some articles such as [1] are devoted to constructing Lift $(f)$ for a particular case of germs.

The purpose of this paper is giving a systematic method to construct liftable vector fields over a multigerm. In order to create the systematic method, we first concentrate on obtaining a reasonable class of multigerms for which the following problems can be affirmatively answered. 
Problem 1. Let $f:\left(\mathbb{K}^{n}, S\right) \rightarrow\left(\mathbb{K}^{p}, 0\right)$ be an analytic multigerm.

(1) Is the module of vector fields liftable over $f$ finitely generated ?

(2) Can we characterize the minimal number of generators when the module of vector fields liftable over $f$ is finitely generated?

(3) Can we calculate the minimal number of generators when the module of vector fields liftable over $f$ is finitely generated ?

(4) Can we construct generators when the module of vector fields liftable over $f$ is finitely generated?

In order to obtain such a reasonable class, we generalize Mather's homomorphism ([15])

$$
\bar{\omega} f: \frac{\theta_{0}(p)}{m_{0} \theta_{0}(p)} \rightarrow \frac{\theta_{S}(f)}{T \mathcal{K}_{e}(f)}
$$

defined by $\bar{\omega} f([\xi])=[\omega f(\xi)]$. Notice that

$$
\frac{\theta_{S}(f)}{T \mathcal{K}_{e}(f)} \cong \frac{\frac{\theta_{S}(f)}{T \mathcal{R}_{e}(f)}}{f^{*} m_{0}\left(\frac{\theta_{S}(f)}{T \mathcal{R}_{e}(f)}\right)}
$$

as finite dimensional vector spaces over $\mathbb{K}$ for any analytic multigerm $f$ satisfying $\operatorname{dim}_{\mathbb{K}} \theta_{S}(f) / T \mathcal{K}_{e}(f)<\infty$. Thus, by the preparation theorem (for instance, see [2]), we have that $\theta_{S}(f)=T \mathcal{A}_{e}(f)$ if and only if $\bar{\omega} f$ is surjective for any analytic multigerm $f$ satisfying $\operatorname{dim}_{\mathbb{K}} \theta_{S}(f) / T \mathcal{K}_{e}(f)<\infty$. In the case that $\mathbb{K}=\mathbb{C}, n \geq p$ and $S=\{$ one point $\}$, the map $\hat{\omega} f: \theta_{0}(p) \rightarrow \frac{\theta_{S}(f)}{T \mathcal{R}_{e}(f)}$ given by $\hat{\omega} f(\xi)=[\omega f(\xi)]$ is called the Kodaira-Spencer map of $f$ and Mather's homomorphism $\bar{\omega} f$ is called the reduced Kodaira-Spencer map of $f([13)$. Thus, $\bar{\omega} f$, which we call the reduced Kodaira-Spencer-Mather map, is a generalization of the reduced Kodaira-Spencer map of $f$; and the module of vector fields liftable over $f$ is the kernel of $\hat{\omega} f$. We would like to have higher versions of $\bar{\omega} f$. For a non-negative integer $i$, an element of $m_{S}^{i}$ or $m_{0}^{i}$ is a germ of analytic function such that the terms of the Taylor series of it up to $(i-1)$ are zero. Thus, $m_{S}^{0}=C_{S}$ and $m_{0}^{0}=C_{0}$. For any non-negative integer $i$ and a given analytic multigerm $f:\left(\mathbb{K}^{n}, S\right) \rightarrow\left(\mathbb{K}^{p}, 0\right)$, we let

$$
{ }_{i} \bar{\omega} f: \frac{m_{0}^{i} \theta_{0}(p)}{m_{0}^{i+1} \theta_{0}(p)} \rightarrow \frac{f^{*} m_{0}^{i} \theta_{S}(f)}{T \mathcal{R}_{e}(f) \cap f^{*} m_{0}^{i} \theta_{S}(f)+f^{*} m_{0}^{i+1} \theta_{S}(f)}
$$

be a homomorphism of $C_{0}$-modules via $f$ defined by ${ }_{i} \bar{\omega} f([\xi])=[\omega f(\xi)]$. Then, ${ }_{i} \bar{\omega} f$ is clearly well-defined. In this paper, we call ${ }_{i} \bar{\omega} f$ a higher version of reduced Kodaira-Spencer-Mather map. Note that ${ }_{0} \bar{\omega} f=\bar{\omega} f$. Similarly as the target module of $\bar{\omega} f$, for any non-negative integer $i$ and any analytic multigerm $f$ satisfying $\operatorname{dim}_{\mathbb{K}} \theta_{S}(f) / T \mathcal{K}_{e}(f)<\infty$, the target module of ${ }_{i} \bar{\omega} f$ is isomorphic to the following:

$$
\frac{\frac{f^{*} m_{0}^{i} \theta_{S}(f)}{T_{\mathcal{R}}(f) \cap f^{*} m_{0}^{i} \theta_{S}(f)}}{f^{*} m_{0}\left(\frac{f^{*} m_{0}^{i} \theta_{S}(f)}{T \mathcal{R}_{e}(f) \cap f^{*} m_{0}^{i} \theta_{S}(f)}\right)} .
$$

Thus, again by the preparation theorem, we have that $f^{*} m_{0}^{i} \theta_{S}(f) \subset T \mathcal{A}_{e}(f)$ if and only if $i \bar{\omega} f$ is surjective. The following clearly holds:

Lemma 1.1. Let $f:\left(\mathbb{K}^{n}, S\right) \rightarrow\left(\mathbb{K}^{p}, 0\right)$ be an analytic multigerm satisfying the condition $\operatorname{dim}_{\mathbb{K}} \theta_{S}(f) / T \mathcal{K}_{e}(f)<\infty$. Then, the following hold: 
(1) Suppose that there exists a non-negative integer $i$ such that ${ }_{i} \bar{\omega} f$ is surjective. Then, $j \bar{\omega} f$ is surjective for any integer $j$ such that $i<j$.

(2) Suppose that there exists a non-negative integer $i$ such that ${ }_{i} \bar{\omega} f$ is injective. Then, $j \bar{\omega} f$ is injective for any non-negative integer $j$ such that $i>j$.

Definition 1.1. Let $f:\left(\mathbb{K}^{n}, S\right) \rightarrow\left(\mathbb{K}^{p}, 0\right)$ be an analytic multigerm satisfying the condition $\operatorname{dim}_{\mathbb{K}} \theta_{S}(f) / T \mathcal{K}_{e}(f)<\infty$.

(1) Set $I_{1}(f)=\left\{i \in\{0\} \cup \mathbb{N} \mid{ }_{i} \bar{\omega} f\right.$ is surjective $\}$. Define $i_{1}(f)$ as

$$
i_{1}(f)=\left\{\begin{array}{cc}
\infty & \left(\text { if } I_{1}(f)=\emptyset\right) \\
\min I_{1}(f) & \left(\text { if } I_{1}(f) \neq \emptyset\right) .
\end{array}\right.
$$

(2) Set $I_{2}(f)=\left\{i \in\{0\} \cup \mathbb{N} \mid{ }_{i} \bar{\omega} f\right.$ is injective $\}$. Define $i_{2}(f)$ as

$$
i_{2}(f)=\left\{\begin{array}{cc}
-\infty & \left(\text { if } I_{2}(f)=\emptyset\right) \\
\max I_{2}(f) & \left(\text { if } \emptyset \neq I_{2}(f) \neq\{0\} \cup \mathbb{N}\right) \\
\infty & \left(\text { if } I_{2}(f)=\{0\} \cup \mathbb{N}\right) .
\end{array}\right.
$$

An analytic multigerm $f:\left(\mathbb{K}^{n}, S\right) \rightarrow\left(\mathbb{K}^{p}, 0\right)$ is said to be finitely determined if there exists a positive integer $k$ such that the inclusion $m_{S}^{k} \theta_{S}(f) \subset T \mathcal{A}_{e}(f)$ holds. The proof of the assertion (ii) of proposition 4.5.2 in [28] works well to show the following:

Proposition 1. Let $f:\left(\mathbb{K}^{n}, S\right) \rightarrow\left(\mathbb{K}^{p}, 0\right)$ be a finitely determined multigerm satisfying $\theta_{S}(f) \neq T \mathcal{A}_{e}(f)$. Then, $i_{2}(f) \geq 0$.

From here we concentrate on dealing with the case $n \leq p$ because the purpose of this paper is to construct liftable vector fields over a multigerm with finite multiplicity. However, the last section is devoted to extending the results to the case $n>p$. Suppose that $f:\left(\mathbb{K}^{n}, S\right) \rightarrow\left(\mathbb{K}^{p}, 0\right)$ is finitely determined. Then, since it is clear that $f^{*} m_{0} C_{S} \subset m_{S}$, there exists a positive integer $k$ such that the inclusion $f^{*} m_{0}^{k} \theta_{S}(f) \subset T \mathcal{A}_{e}(f)$ holds. Thus, $k \bar{\omega} f$ is surjective. Conversely, suppose that there exists a positive integer $k$ such that ${ }_{k} \bar{\omega} f$ is surjective for an analytic multigerm $f:\left(\mathbb{K}^{n}, S\right) \rightarrow\left(\mathbb{K}^{p}, 0\right)$ satisfying $\operatorname{dim}_{\mathbb{K}} \theta_{S}(f) / T \mathcal{K}_{e}(f)<\infty$. Then, as we have already confirmed, the inclusion $f^{*} m_{0}^{k} \theta_{S}(f) \subset T \mathcal{A}_{e}(f)$ holds by the preparation theorem. In the case $n \leq p$, by Wall's estimate (theorem 4.6.2 in [28]), the condition $\operatorname{dim}_{\mathbb{K}} \theta_{S}(f) / T \mathcal{K}_{e}(f)<\infty$ implies that there exists an integer $\ell$ such that $m_{S}^{\ell} \subset f^{*} m_{0} C_{S}$. Hence, we have the following:

Proposition 2. Let $f:\left(\mathbb{K}^{n}, S\right) \rightarrow\left(\mathbb{K}^{p}, 0\right)$ be an analytic multigerm satisfying the condition $\operatorname{dim}_{\mathbb{K}} \theta_{S}(f) / T \mathcal{K}_{e}(f)<\infty$. Suppose that $n \leq p$. Then, $i_{1}(f)<\infty$ if and only if $f$ is finitely determined.

An analytic multigerm $f:\left(\mathbb{K}^{n}, S\right) \rightarrow\left(\mathbb{K}^{p}, 0\right)(n \leq p)$ is said to be of corank at most one if $\max \left\{n-\operatorname{rank} J f\left(s_{j}\right)|1 \leq j \leq| S \mid\right\} \leq 1$ holds, where $\operatorname{Jf}\left(s_{j}\right)$ is the Jacobian matrix of $f$ at $s_{j} \in S$ and $|S|$ stands for the number of distinct points of $S$.

Proposition 3. Let $f:\left(\mathbb{K}^{n}, S\right) \rightarrow\left(\mathbb{K}^{p}, 0\right)(n \leq p)$ be a finitely determined multigerm of corank at most one. Then, $i_{1}(f) \geq i_{2}(f)$.

Proposition 3 is proved in 92 Proposition 3 yields the following corollary.

Corollary 1. Let $f:\left(\mathbb{K}^{n}, S\right) \rightarrow\left(\mathbb{K}^{p}, 0\right)(n \leq p)$ be a finitely determined multigerm of corank at most one. Suppose that there exists a non-negative integer $i$ such that $i_{1}(f)=i_{2}(f)=i$. Then, the following hold: 
(1) For any non-negative integer $j$ such that $j<i,{ }_{j} \bar{\omega} f$ is injective but not surjective.

(2) For any non-negative integer $j$ such that $i<j,{ }_{j} \bar{\omega} f$ is surjective but not injective.

Example 1.1. Let $e: \mathbb{K} \rightarrow \mathbb{K}^{2}$ be the embedding defined by $e(x)=(x, 0)$ and for any real number $\theta$ let $R_{\theta}: \mathbb{K}^{2} \rightarrow \mathbb{K}^{2}$ be the linear map which gives the rotation of $\mathbb{K}^{2}$ about the origin with respect to the angle $\theta$.

$$
R_{\theta}\left(\begin{array}{c}
X \\
Y
\end{array}\right)=\left(\begin{array}{rr}
\cos \theta & -\sin \theta \\
\sin \theta & \cos \theta
\end{array}\right)\left(\begin{array}{l}
X \\
Y
\end{array}\right) .
$$

For any non-negative integer $\ell$ set $S=\left\{s_{0}, \ldots, s_{\ell+1}\right\}\left(s_{j} \neq s_{k}\right.$ if $\left.j \neq k\right)$. Define $\theta_{j}=j \frac{\pi}{\ell+2}$ and set $e_{j}:\left(\mathbb{K}, s_{j}\right) \rightarrow\left(\mathbb{K}^{2}, 0\right)$ as $e_{j}\left(x_{j}\right)=R_{\theta_{j}} \circ e\left(x_{j}\right)$ for any $j(0 \leq j \leq$ $\ell+1)$, where $x_{j}=x-s_{j}$. Then, $E_{\ell}=\left\{e_{0}, \ldots, e_{\ell+1}\right\}:(\mathbb{K}, S) \rightarrow\left(\mathbb{K}^{2}, 0\right)$ is a finitely determined multigerm of corank at most one. The image of $E_{\ell}$ is a line arrangement and hence the Euler vector field of the defining equation of the image of $E_{\ell}$ is a liftable vector field over $E_{\ell}$. It follows that ${ }_{1} \bar{\omega} E_{\ell}$ is not injective. Furthermore, it is easily seen that ${ }_{0} \bar{\omega} E_{\ell}$ is injective even in the case $\ell=0$ (in the case $\ell \geq 1$ this is a trivial corollary of Proposition (1). Thus, $i_{2}\left(E_{\ell}\right)=0$. On the other hand, it is not hard to show that $i_{1}\left(E_{\ell}\right)=\ell$. Therefore, $i_{1}\left(E_{\ell}\right)-i_{2}\left(E_{\ell}\right)=\ell$.

This example shows that, in general, there are no upper bounds of $i_{1}(f)-i_{2}(f)$ for a finitely determined multigerm $f:\left(\mathbb{K}^{n}, S\right) \rightarrow\left(\mathbb{K}^{p}, 0\right)(n \leq p)$ of corank at most one. This example shows also that the integer $i_{1}(f)-i_{2}(f)$ measures how well-behaved a given finitely determined multigerm of corank at most one is from the viewpoint of liftable vector fields.

The following Theorem 1 shows that the desired reasonable class is the set consisting of finitely determined multigerms $f:\left(\mathbb{K}^{n}, S\right) \rightarrow\left(\mathbb{K}^{p}, 0\right)(n \leq p)$ of corank at most one satisfying $i_{1}(f)=i_{2}(f)$.

Theorem 1. Let $f:\left(\mathbb{K}^{n}, S\right) \rightarrow\left(\mathbb{K}^{p}, 0\right)(n \leq p)$ be a finitely determined multigerm of corank at most one. Suppose that there exists a non-negative integer $i$ such that $i_{1}(f)=i_{2}(f)=i$. Then, the minimal number of generators for the module of vector fields liftable over $f$ is exactly $\operatorname{dim}_{\mathbb{K}} \operatorname{ker}\left({ }_{i+1} \bar{\omega} f\right)$.

Notice that the embedding $e$ in Example 1.1 does not satisfy the assumption of Theorem 11. Actually, since $0 \bar{\omega} e$ is surjective but not injective, $i_{1}(e)=0$ and $i_{2}(e)=-\infty$. On the other hand, the multigerm $E_{0}$ in Example 1.1 does satisfy the assumption of Theorem 1 though $E_{0}$ does not satisfy the assumption of Proposition 1. Furthermore, a lot of examples of Theorem 1 are given by Proposition 4 (see also Section 3).

Definition 1.2. (1) A multigerm $f:\left(\mathbb{K}^{n}, S\right) \rightarrow\left(\mathbb{K}^{p}, 0\right)$ is said to be stable if it satisfies $\theta_{S}(f)=T_{e} \mathcal{A}(f)$.

(2) Define the mapping ev $: v_{0}(p) \rightarrow T_{0}\left(\mathbb{R}^{p}\right)$ by $e v_{0}(\eta)=\eta(0)$.

(3) A stable multigerm $f:\left(\mathbb{K}^{n}, S\right) \rightarrow\left(\mathbb{K}^{p}, 0\right)$ is said to be isolated if $e v_{0}(\eta)=0$ for any $\eta \in \operatorname{Lift}(f)$.

The following proposition shows that our reasonable class contains the set consisting of isolated stable multigerms $f:\left(\mathbb{K}^{n}, S\right) \rightarrow\left(\mathbb{K}^{p}, 0\right)(n \leq p)$ of corank at most one.

Proposition 4. Let $f:\left(\mathbb{K}^{n}, S\right) \rightarrow\left(\mathbb{K}^{p}, 0\right)(n \leq p)$ be a finitely determined multigerm of corank at most one. Then, the following hold: 
(1) In the case $i=0$, the following hold:

(a) ${ }_{0} \bar{\omega} f$ is surjective if and only if $f$ is stable.

(b) $0 \bar{\omega} f$ is injective if and only if $f$ is isolated.

(2) In the case $i=1$, the following hold:

(a) ${ }_{1} \bar{\omega} f$ is surjective if and only if $T \mathcal{A}(f)=T \mathcal{K}(f)$.

(b) ${ }_{1} \bar{\omega} f$ is injective if only if for any $\eta \in$ Lift $(f) \eta$ has no constant terms and no linear terms. Moreover, these equivalent conditions imply that $\operatorname{dim}_{\mathbb{K}} \theta_{S}(f) / T \mathcal{A}_{e}(f)>1$.

Next, in order to answer (3) of Problem 1 for a given finitely determined multigerm $f:\left(\mathbb{K}^{n}, S\right) \rightarrow\left(\mathbb{K}^{p}, 0\right)(n \leq p)$ of corank at most one such that $0 \leq i_{1}(f)=$ $i_{2}(f)<\infty$, we generalize Wall's homomorphism $([28])$

$$
\bar{t} f: Q(f)^{n} \rightarrow Q(f)^{p}, \quad \bar{t} f([\eta])=[t f(\eta)]
$$

as follows. For a given analytic multigerm $f:\left(\mathbb{K}^{n}, S\right) \rightarrow\left(\mathbb{K}^{p}, 0\right)$ satisfying the condition $\operatorname{dim}_{\mathbb{K}} Q(f)<\infty$, let $\delta(f)$ (resp., $\gamma(f)$ ) be the dimension of the vector space $Q(f)$ (resp., the dimension of the kernel of $\bar{t} f$ ). For the $f$ and a non-negative integer $i$, we Set ${ }_{i} Q(f)=f^{*} m_{0}^{i} C_{S} / f^{*} m_{0}^{i+1} C_{S}$ and ${ }_{i} \delta(f)=\operatorname{dim}_{\mathbb{K}} i Q(f)$. Thus, we have that ${ }_{0} Q(f)=Q(f)$ and ${ }_{0} \delta(f)=\delta(f)=\operatorname{dim}_{\mathbb{K}} Q(f)$. The $Q(f)$-modules ${ }_{i} Q(f)^{n}$ and ${ }_{i} Q(f)^{p}$ may be identified with the following respectively.

$$
\frac{f^{*} m_{0}^{i} \theta_{S}(n)}{f^{*} m_{0}^{i+1} \theta_{S}(n)} \text { and } \frac{f^{*} m_{0}^{i} \theta_{S}(f)}{f^{*} m_{0}^{i+1} \theta_{S}(f)}
$$

Let ${ }_{i} \gamma(f)$ be the dimension of the kernel of the following well-defined homomorphism of $Q(f)$-modules.

$$
{ }_{i} \bar{t} f:{ }_{i} Q(f)^{n} \rightarrow{ }_{i} Q(f)^{p}, \quad{ }_{i} \bar{t} f([\eta])=[t f(\eta)] .
$$

Then, we have that ${ }_{i} \delta(f)<\infty$ if $\delta(f)<\infty$ and ${ }_{i} \gamma(f)<\infty$ if $\gamma(f)<\infty$. For details on ${ }_{i} Q(f),{ }_{i} \delta(f),{ }_{i} \bar{t} f$ and ${ }_{i} \gamma(f)$, see [21].

Proposition 5. Let $f:\left(\mathbb{K}^{n}, S\right) \rightarrow\left(\mathbb{K}^{p}, 0\right)$ be an analytic multigerm with finite multiplicity and of corank at most one. Suppose that there exists a non-negative integer $i$ such that ${ }_{i+1} \bar{\omega} f$ is surjective. Then, the following holds:

$$
\operatorname{dim}_{\mathbb{K}} \operatorname{ker}(i+1 \bar{\omega} f)=p \cdot\left(\begin{array}{c}
p+i \\
i+1
\end{array}\right)-\left((p-n) \cdot{ }_{i+1} \delta(f)+{ }_{i+1} \gamma(f)-{ }_{i} \gamma(f)\right)
$$

where the dot in the center stands for the multiplication.

Proposition 6. Let $f:\left(\mathbb{K}^{n}, S\right) \rightarrow\left(\mathbb{K}^{p}, 0\right)$ be an analytic multigerm with finite multiplicity and of corank at most one. Then, the following hold:

(1) $0 \gamma(f)=\gamma(f)=\delta(f)-|S|$.

$$
{ }_{i} \delta(f)=\left(\begin{array}{c}
n+i-1 \\
i
\end{array}\right) \cdot \delta(f),{ }_{i} \gamma(f)=\left(\begin{array}{c}
n+i-1 \\
i
\end{array}\right) \cdot \gamma(f) \quad(i \in \mathbb{N} \cup\{0\}) .
$$

By combining Propositions 5 and 6, for an analytic multigerm $f$ of corank at most one such that $\operatorname{dim}_{\mathbb{K}} Q(f)<\infty$, the $\mathcal{A}$-invariant " $\operatorname{dim}_{\mathbb{K}} \operatorname{ker}\left({ }_{i+1} \bar{\omega} f\right)$ " can be calculated easily by using $\mathcal{K}$-invariants " $\delta(f), \gamma(f)$ " when there exists a non-negative integer $i$ such that $i+1 \bar{\omega} f$ is surjective.

Theorem 2. Let $f:\left(\mathbb{K}^{n}, S\right) \rightarrow\left(\mathbb{K}^{p}, 0\right)$ be an analytic multigerm with finite multiplicity and of corank at most one. 
(1) Let $F:\left(\mathbb{K}^{n} \times \mathbb{K}^{r}, S \times\{0\}\right) \rightarrow\left(\mathbb{K}^{p} \times \mathbb{K}^{r},(0,0)\right)$ be a stable unfolding of $f$. Let $\eta=\left(\eta_{1}, \ldots, \eta_{p}, \eta_{p+1}, \ldots, \eta_{p+r}\right)$ be an element of the intersection $\operatorname{Lift}(F) \cap$ Lift $(g)$, where $g=\left\{g_{1}, \ldots, g_{r}\right\}$ with $g_{i}:\left(\mathbb{K}^{p} \times \mathbb{K}^{r},(0,0)\right) \rightarrow\left(\mathbb{K}^{p} \times \mathbb{K}^{r},(0,0)\right)$ defined by $g_{i}\left(X_{1}, \ldots, X_{p}, \lambda_{1}, \ldots, \lambda_{r}\right)=\left(X_{1}, \ldots, X_{p}, \lambda_{1}, \ldots, \lambda_{i-1}, \lambda_{i}^{2}, \lambda_{i+1}, \ldots, \lambda_{r}\right)$ $(1 \leq i \leq r)$. Then, $\bar{\eta}(X)=\left(\eta_{1}(X, 0), \ldots, \eta_{p}(X, 0)\right)$ is an element of Lift $(f)$.

(2) Suppose that $f$ admits a one-parameter stable unfolding $F:\left(\mathbb{K}^{n} \times \mathbb{K}, S \times\right.$ $\{0\}) \rightarrow\left(\mathbb{K}^{p} \times \mathbb{K},(0,0)\right)$. Then, for any $\bar{\eta} \in$ Lift $(f)$ there exists an element $\eta=\left(\eta_{1}, \ldots, \eta_{p}, \eta_{p+1}\right) \in \operatorname{Lift}(F) \cap \operatorname{Lift}\left(g_{1}\right)$ such that the equality $\bar{\eta}(X)=$ $\left(\eta_{1}(X, 0), \ldots, \eta_{p}(X, 0)\right)$ holds.

The proof of Theorem 1 provides a recipe for constructing all liftable vector fields over a finitely determined multigerm $f$ of corank at most one satisfying $i_{1}(f)=$ $i_{2}(f)$. In particular, by Proposition 4, all liftable vector fields over an isolated stable multigerm $f$ of corank at most one can be constructed. Since any stable germ is $\mathcal{A}$-equivalent to a prism on an isolated stable multigerm, all liftable vector fields over any stable germ can be constructed. Moreover, by using Theorem 2, we can construct all liftable vector fields over an analytic multigerm $f$ of corank at most one admitting a one-parameter stable unfolding $F$ from $\operatorname{Lift}(F)$. It is clear also that if $f$ satisfies $\operatorname{dim}_{\mathbb{K}} \theta_{S}(f) / T \mathcal{A}_{e}(f)=1$ (namely, $f$ is a multigerm of $\mathcal{A}_{e^{-}}$ codimension one), then $f$ admits a one-parameter stable unfolding. Thus, we can construct all liftable vector fields over a multigerm of corank at most one and of $\mathcal{A}_{e}$-codimension one. In particular, for any augmentation defined in [5], all liftable vector fields over it can be constructed by our recipe. In Remark 1 at the end of Section 6 an idea on how big the space of germs which admit a one-parameter stable unfolding is is given.

This paper is organized as follows. In Section 2, proofs of Propositions 3, 4, 5, and 6 are given. In Section 3. examples for which actual calculations of minimal numbers of generators are carried out are given. Theorem 1 (resp., Theorem 2) is proved in Sections 4 (resp., Section [5). In Section 6 , by constructing concrete generators for several examples using Theorem 2 and the proof of Theorem [1] it is explained in detail how to construct liftable vector fields over an analytic multigerm of corank at most one admitting a one-parameter stable unfolding. Finally, Section 7 generalizes the results for the case $n>p$.

\section{Proofs of Propositions 3, 4, 5, and 6]}

Firstly, Proposition [6 is proved.

Proof of Proposition 6. 
Set $S=\left\{s_{1}, \ldots, s_{|S|}\right\}\left(s_{j} \neq s_{k}\right.$ if $\left.j \neq k\right)$ and for any $j(1 \leq j \leq|S|)$ let $f_{j}$ be the restriction $\left.f\right|_{\left(\mathbb{K}^{n}, s_{j}\right)}$. Then, we have the following:

$$
\begin{aligned}
\delta(f)=\operatorname{dim}_{\mathbb{K}} Q(f)=\sum_{j=1}^{|S|} \operatorname{dim}_{\mathbb{K}} Q\left(f_{j}\right) & =\sum_{j=1}^{|S|} \delta\left(f_{j}\right) . \\
\gamma(f)=\operatorname{dim}_{\mathbb{K}} \operatorname{ker}(\bar{t} f)=\sum_{j=1}^{|S|} \operatorname{dim}_{\mathbb{K}} \operatorname{ker}\left(\bar{t} f_{j}\right) & =\sum_{j=1}^{|S|} \gamma\left(f_{j}\right) \\
& =\sum_{j=1}^{|S|}\left(\delta\left(f_{j}\right)-1\right)=\delta(f)-|S| .
\end{aligned}
$$

This completes the proof of the assertion 1 of Proposition 6

Next we prove the assertion 2 of Proposition [6. Since $f$ is of corank at most one, for any $j(1 \leq j \leq|S|)$ there exist germs of diffeomorphism $h_{j}:\left(\mathbb{K}^{n}, s_{j}\right) \rightarrow\left(\mathbb{K}^{n}, s_{j}\right)$ and $H_{j}:\left(\mathbb{K}^{p}, 0\right) \rightarrow\left(\mathbb{K}^{p}, 0\right)$ such that $H_{j} \circ f_{j} \circ h_{j}^{-1}$ has the following form:

$$
H_{j} \circ f_{j} \circ h_{j}^{-1}(x, y)=\left(x, y^{\delta\left(f_{j}\right)}+f_{j, n}(x, y), f_{j, n+1}(x, y), \ldots, f_{j, p}(x, y)\right) .
$$

Here, $(x, y)=\left(x_{1}, \ldots, x_{n-1}, y\right)$ is the local coordinate with respect to the coordinate neighborhood $\left(U_{j}, h_{j}\right)$ at $s_{j}$ and $f_{j, q}$ satisfies $f_{j, q}(0, \ldots, 0, y)=o\left(y^{\delta\left(f_{j}\right)}\right)$ for any $q$ $(n \leq q \leq p)$. By the preparation theorem, $C_{s_{j}}$ is generated by $1, y, \ldots, y^{\delta\left(f_{j}\right)-1}$ as $C_{0}$-module via $f_{j}$. Thus, $f_{j}^{*} m_{0}^{i} C_{s_{j}}$ is generated by elements of the following set as $C_{0}$-module via $f_{j}$.

$$
\left\{x_{1}^{k_{1}} \cdots x_{n-1}^{k_{n-1}} y^{k_{n} \delta\left(f_{j}\right)+\ell} \mid k_{m} \geq 0, \sum_{m=1}^{n} k_{m}=i, 0 \leq \ell \leq \delta\left(f_{j}\right)-1\right\} .
$$

Thus, the following set is a basis of $i Q\left(f_{j}\right)$.

$$
\left\{\left[x_{1}^{k_{1}} \cdots x_{n-1}^{k_{n-1}} y^{k_{n} \delta\left(f_{j}\right)+\ell}\right] \mid k_{m} \geq 0, \sum_{m=1}^{n} k_{m}=i, 0 \leq \ell \leq \delta\left(f_{j}\right)-1\right\} .
$$

Therefore, we have the following:

$$
\begin{aligned}
{ }_{i} \delta(f)=\operatorname{dim}_{\mathbb{K} i} Q(f)=\sum_{j=1}^{|S|} \operatorname{dim}_{\mathbb{K} i} Q\left(f_{j}\right) & =\sum_{j=1}^{|S|} \operatorname{dim}_{\mathbb{K}} \frac{f_{j}^{*} m_{0}^{i} C_{s_{j}}}{f_{j}^{*} m_{0}^{i+1} C_{s_{j}}} \\
& =\sum_{j=1}^{|S|}\left(\begin{array}{c}
n+i-1 \\
i
\end{array}\right) \cdot \delta\left(f_{j}\right) \\
& =\left(\begin{array}{c}
n+i-1 \\
i
\end{array}\right) \cdot \sum_{j=1}^{|S|} \delta\left(f_{j}\right) \\
& =\left(\begin{array}{c}
n+i-1 \\
i
\end{array}\right) \cdot \delta(f) .
\end{aligned}
$$

Next we prove the formula for $i \gamma(f)$. Since it is clear that ${ }_{i} \gamma\left(f_{j}\right)$ does not depend on the particular choice of coordinate systems of $\left(\mathbb{K}^{n}, s_{j}\right)$ and of $\left(\mathbb{K}^{p}, 0\right)$, we may 
assume that $f_{j}$ has the above form from the first. Then, it is easily seen that the following set is a basis of $\operatorname{ker}_{i} \bar{t} f_{j}$.

$$
\left\{[\underbrace{0 \oplus \cdots \oplus 0}_{(n-1) \text { tuples }} \oplus x_{1}^{k_{1}} \cdots x_{n-1}^{k_{n-1}} y^{k_{n} \delta\left(f_{j}\right)+\ell}] \mid k_{m} \geq 0, \sum_{m=1}^{n} k_{m}=i, 1 \leq \ell \leq \delta\left(f_{j}\right)-1\right\} .
$$

Therefore, we have the following:

$$
\begin{aligned}
{ }_{i} \gamma(f)=\operatorname{dim}_{\mathbb{K}} \operatorname{ker}\left({ }_{i} \bar{t} f\right)=\sum_{j=1}^{|S|} \operatorname{dim}_{\mathbb{K}} \operatorname{ker}\left({ }_{i} \bar{t} f_{j}\right) & =\sum_{j=1}^{|S|}\left(\begin{array}{c}
n+i-1 \\
i
\end{array}\right) \cdot\left(\delta\left(f_{j}\right)-1\right) \\
& =\left(\begin{array}{c}
n+i-1 \\
i
\end{array}\right) \cdot \sum_{j=1}^{|S|} \gamma\left(f_{j}\right) \\
& =\left(\begin{array}{c}
n+i-1 \\
i
\end{array}\right) \cdot \gamma(f) .
\end{aligned}
$$

Q.E.D.

Secondly, Proposition 5 is proved.

Proof of Proposition 5

Consider the linear map ${ }_{i+1} \bar{t} f$. Then, we have the following:

$$
\operatorname{dim}_{\mathbb{K} i+1} Q(f)^{n}={ }_{i+1} \gamma(f)+\operatorname{dim}_{\mathbb{K}} \operatorname{Image}\left({ }_{i+1} \bar{t} f\right) .
$$

Since $\operatorname{dim}_{\mathbb{K}} Q(f)<\infty$ and $f$ is of corank at most one, it is easily seen that $t f$ is injective. Hence we see that

$$
\operatorname{dim}_{\mathbb{K}} \frac{T \mathcal{R}_{e}(f) \cap f^{*} m_{0}^{i+1} \theta_{S}(f)}{T \mathcal{R}_{e}(f) \cap f^{*} m_{0}^{i+2} \theta_{S}(f)}=\operatorname{dim}_{\mathbb{K}} \operatorname{Image}\left({ }_{i+1} \bar{t} f\right)+{ }_{i} \gamma(f) .
$$

Therefore, we have the following:

$\operatorname{dim}_{\mathbb{K}} \frac{f^{*} m_{0}^{i+1} \theta_{S}(f)}{T \mathcal{R}_{e}(f) \cap f^{*} m_{0}^{i+1} \theta_{S}(f)+f^{*} m_{0}^{i+2} \theta_{S}(f)}=(p-n) \cdot{ }_{i+1} \delta(f)+{ }_{i+1} \gamma(f)-{ }_{i} \gamma(f)$.

Hence, we have the following:

$$
\begin{aligned}
& \operatorname{dim}_{\mathbb{K}} \operatorname{ker}(i+1 \bar{\omega} f) \\
= & \operatorname{dim}_{\mathbb{K}} \frac{m_{0}^{i+1} \theta_{0}(p)}{m_{0}^{i+2} \theta_{0}(p)}-\operatorname{dim}_{\mathbb{K}} \frac{f^{*} m_{0}^{i+1} \theta_{S}(f)}{T \mathcal{R}_{e}(f) \cap f^{*} m_{0}^{i+1} \theta_{S}(f)+f^{*} m_{0}^{i+2} \theta_{S}(f)} \\
= & p \cdot\left(\begin{array}{c}
p+i \\
i+1
\end{array}\right)-\left((p-n) \cdot{ }_{i+1} \delta(f)+{ }_{i+1} \gamma(f)-{ }_{i} \gamma(f)\right) .
\end{aligned}
$$

Q.E.D.

Thirdly, Proposition 3 is proved.

Proof of Proposition 3

By Lemma [1.1] it sufficies to show that for any $i$ and any finitely determined multigerm $f:\left(\mathbb{K}^{n}, S\right) \rightarrow\left(\mathbb{K}^{p}, 0\right)(n \leq p)$ of corank at most one satisfying that $i \bar{\omega} f$ is surjective, ${ }_{i+1} \bar{\omega} f$ is not injective. By Lemma 1.1 ${ }_{i+1} \bar{\omega} f$ is not injective if ${ }_{i} \bar{\omega} f$ is not injective. Thus, we may assume that ${ }_{i} \bar{\omega} f$ is bijective. 
We first prove Proposition 3 in the case $i=0$. Since we have assumed that ${ }_{0} \bar{\omega} f$ is bijective, the following holds (see [17] or [28]):

$$
p \cdot\left(\begin{array}{c}
p-1 \\
0
\end{array}\right)=\operatorname{dim}_{\mathbb{K}} \frac{\theta_{0}(p)}{m_{0} \theta_{0}(p)}=(p-n) \cdot{ }_{0} \delta(f)+{ }_{0} \gamma(f) .
$$

Note that the above equality can not be obtained by Proposition 5 Note further that at least one of $p-n>0$ or $0 \gamma(f)>0$ holds by this equality. We have the following:

$$
\begin{aligned}
p \cdot\left(\begin{array}{l}
p \\
1
\end{array}\right) & =p^{2} \cdot\left(\begin{array}{c}
p-1 \\
0
\end{array}\right) \\
& =p \cdot\left((p-n) \cdot{ }_{0} \delta(f)+{ }_{0} \gamma(f)\right) \\
& =\frac{p}{n} \cdot\left((p-n) \cdot{ }_{1} \delta(f)+{ }_{1} \gamma(f)\right) \quad(\text { by } 2 \text { of Proposition } 5) \\
& \geq(p-n) \cdot{ }_{1} \delta(f)+{ }_{1} \gamma(f) \quad(\text { by } n \leq p) \\
& \geq(p-n) \cdot{ }_{1} \delta(f)+{ }_{1} \gamma(f)-{ }_{0} \gamma(f) \quad\left(\text { by }{ }_{0} \gamma(f) \geq 0\right) .
\end{aligned}
$$

Since we have confirmed that at least one of $p-n>0$ or ${ }_{0} \gamma(f)>0$ holds, we have the following sharp inequality:

$$
p \cdot\left(\begin{array}{c}
p \\
1
\end{array}\right)>(p-n) \cdot{ }_{1} \delta(f)+{ }_{1} \gamma(f)-{ }_{0} \gamma(f)
$$

Hence ${ }_{1} \bar{\omega} f$ is not injective by Lemma 1.1 and Proposition 5 .

Next we prove Proposition 3 in the case $i \geq 1$. Since we have assumed that ${ }_{i} \bar{\omega} f$ is bijective, we have the following equality by Proposition 5 :

$$
\begin{aligned}
& p \cdot\left(\begin{array}{c}
p+i-1 \\
i
\end{array}\right) \\
= & (p-n) \cdot{ }_{i} \delta(f)+{ }_{i} \gamma(f)-{ }_{i-1} \gamma(f) \\
= & \left.(p-n) \cdot{ }_{i} \delta(f)+\left(1-\frac{i}{n+i-1}\right) \cdot{ }_{i} \gamma(f) \quad \text { (by } 2 \text { of Proposition } 6\right) \\
= & (p-n) \cdot{ }_{i} \delta(f)+\frac{n-1}{n+i-1} \cdot{ }_{i} \gamma(f) .
\end{aligned}
$$


Note that at least one of $p-n>0$ or $(n-1) \cdot{ }_{i} \gamma(f)>0$ holds by this equality. We have the following:

$$
\begin{aligned}
& p \cdot\left(\begin{array}{c}
p+i \\
i+1
\end{array}\right) \\
= & \frac{p+i}{i+1} \cdot p \cdot\left(\begin{array}{c}
p+i-1 \\
i
\end{array}\right) \\
= & \frac{p+i}{i+1} \cdot\left((p-n) \cdot{ }_{i} \delta(f)+\frac{n-1}{n+i-1} \cdot{ }_{i} \gamma(f)\right) \\
= & \frac{p+i}{i+1} \cdot\left(\frac{i+1}{n+i} \cdot(p-n) \cdot{ }_{i+1} \delta(f)+\frac{n-1}{n+i-1} \cdot \frac{i+1}{n+i} \cdot i+1 \gamma(f)\right) \\
= & \frac{p+i}{n+i} \cdot(p-n) \cdot{ }_{i+1} \delta(f)+\frac{p+i}{n+i-1} \cdot \frac{n-1}{n+i} \cdot{ }_{i+1} \gamma(f) \\
\geq & (p-n) \cdot{ }_{i+1} \delta(f)+\frac{p+i}{n+i-1} \cdot \frac{n-1}{n+i} \cdot{ }_{i+1} \gamma(f) \quad(\text { by } n \leq p) \\
\geq & \left.(p-n) \cdot{ }_{i+1} \delta(f)+\frac{n-1}{n+i} \cdot{ }_{i+1} \gamma(f) \quad(\text { by } n \leq p \text { and }(n-1))_{i+1} \gamma(f) \geq 0\right) \\
= & \left.(p-n) \cdot{ }_{i+1} \delta(f)+{ }_{i+1} \gamma(f)-{ }_{i} \gamma(f) \quad \text { (by } 2 \text { of Proposition } 6\right) .
\end{aligned}
$$

Since we have confirmed that at least one of $p-n>0$ or $(n-1) \cdot{ }_{i} \gamma(f)>0$ holds and ${ }_{i+1} \gamma(f)=\frac{n+i}{i+1} \cdot{ }_{i} \gamma(f)$ by the assertion 2 of Proposition 6 we wave the following sharp inequality:

$$
p \cdot\left(\begin{array}{c}
p+i \\
i+1
\end{array}\right)>(p-n) \cdot{ }_{i+1} \delta(f)+{ }_{i+1} \gamma(f)-{ }_{i} \gamma(f) .
$$

Hence, ${ }_{i+1} \bar{\omega} f$ is not injective by Lemma 1.1 and Proposition 5

Q.E.D.

Finally, Proposition 4 is proved.

Proof of Proposition 4

Proof of the assertion (1) of Proposition 4 is as follows. Recall that $0 \bar{\omega} f$ is nothing but Mather's $\bar{\omega} f$ defined in [15]. The assertion (a) has been already shown by Mather (see p.228 of [15). Since $\bar{\omega} f: \theta_{0}(p) / m_{0} \theta_{0}(p) \rightarrow \theta_{S}(f) / T \mathcal{K}_{e}(f)$ is defined by $\bar{\omega} f([\eta])=[\eta \circ f]$, by definition, the injectivity of $\bar{\omega} f$ is equivalent to assert that $e v_{0}(\eta)=\eta(0)=0$ for any $\eta \in \operatorname{Lift}(f)$.

Proof of the assertion (2) of Proposition 4 is as follows. Recall that ${ }_{1} \bar{\omega} f$ is the following mapping defined by $1 \bar{\omega} f([\eta])=[\eta \circ f]$ :

$$
{ }_{1} \bar{\omega} f: \frac{m_{0} \theta_{0}(p)}{m_{0}^{2} \theta_{0}(p)} \rightarrow \frac{f^{*} m_{0} \theta_{S}(f)}{T \mathcal{R}_{e}(f) \cap f^{*} m_{0} \theta_{S}(f)+f^{*} m_{0}^{2} \theta_{S}(f)} .
$$

As we have already confirmed in Section 1, by the preparation theorem, we have that ${ }_{1} \bar{\omega} f$ is surjective if and only if $f^{*} m_{0} \theta_{S}(f) \subset T \mathcal{A}_{e}(f)$. Since $f$ is finitely determined, we can conclude that $f^{*} m_{0} \theta_{S}(f) \subset T \mathcal{A}_{e}(f)$ if and only if $f^{*} m_{0} \theta_{S}(f) \subset T \mathcal{A}(f)$.

For (b) of (2), it is easily seen that injectivity of ${ }_{1} \bar{\omega} f$ is equivalent to assert that $\eta$ has no constant terms and no linear terms for any $\eta \in \operatorname{Lift}(f)$. These equivalent conditions imply that $f$ is not $\mathcal{A}$-equivalent to a quasi-homogeneous multigerm. By [5], this implies that $\operatorname{dim}_{\mathbb{K}} \theta_{S}(f) / T \mathcal{A}_{e}(f)>1$.

Q.E.D. 


\section{Examples of Theorem 1}

Example 3.1. Let $\varphi:\left(\mathbb{K}^{n}, 0\right) \rightarrow\left(\mathbb{K}^{n}, 0\right)$ be the map-germ given by $\varphi\left(x_{1}, \ldots, x_{n-1}\right.$, $y)=\left(x_{1}, \ldots, x_{n-1}, y^{n+1}+\sum_{i=1}^{n-1} x_{i} y^{i}\right)$. Then, it is known that $f$ is an isolated stable mono-germ by [20] or [16]. Thus, by Proposition 4, ${ }_{0} \bar{\omega} \varphi$ is bijective. Therefore, it follows that $i_{1}(\varphi)=i_{2}(\varphi)=0$. By Theorem 10. Lemma 1.1 and Propositions 5, 6] the minimal number of generators for $\operatorname{Lift}(\varphi)$ can be calculated as follows:

$$
\begin{aligned}
& n \cdot\left(\begin{array}{c}
n \\
1
\end{array}\right)-\left((n-n) \cdot{ }_{1} \delta(\varphi)+{ }_{1} \gamma(\varphi)-{ }_{0} \gamma(\varphi)\right) \\
= & n^{2}-((n-n) \cdot n \cdot(n+1)+n \cdot(n+1-1)-(n+1-1)) \\
= & n .
\end{aligned}
$$

It has been verified in [1] that the minimal number of generators for $\operatorname{Lift}(\varphi)$ is exactly $n$ in the complex case.

Example 3.2. Let $\varphi_{k}:\left(\mathbb{K}^{2 k-2}, 0\right) \rightarrow\left(\mathbb{K}^{2 k-1}, 0\right)$ be given by

$$
\begin{aligned}
& \varphi_{k}\left(u_{1}, \ldots, u_{k-2}, v_{1}, \ldots, v_{k-1}, y\right) \\
= & \left(u_{1}, \ldots, u_{k-2}, v_{1}, \ldots, v_{k-1}, y^{k}+\sum_{i=1}^{k-2} u_{i} y^{i}, \sum_{i=1}^{k-1} v_{i} y^{i}\right) .
\end{aligned}
$$

Then, it is known that $f$ is an isolated stable mono-germ by [20] or [16. Thus, by Proposition 4, ${ }_{0} \bar{\omega} \varphi_{k}$ is bijective. Therefore, it follows that $i_{1}\left(\varphi_{k}\right)=i_{2}\left(\varphi_{k}\right)=0$. By Theorem 1, Lemma 1.1 and Propositions 5 , 6. the minimal number of generators for $\operatorname{Lift}\left(\varphi_{k}\right)$ can be calculated as follows:

$$
\begin{aligned}
& (2 k-1) \cdot\left(\begin{array}{c}
2 k-1 \\
1
\end{array}\right)-\left(((2 k-1)-(2 k-2)) \cdot{ }_{1} \delta\left(\varphi_{k}\right)+{ }_{1} \gamma\left(\varphi_{k}\right)-{ }_{0} \gamma\left(\varphi_{k}\right)\right) \\
= & (2 k-1)^{2}-(((2 k-1)-(2 k-2)) \cdot(2 k-2) \cdot k \\
= & 3 k-2 .
\end{aligned}
$$

It has been verified in 9 that the minimal number of generators for $\operatorname{Lift}\left(\varphi_{k}\right)$ is exactly $3 k-2$ in the complex case and in the case a set of generators has been obtained in [1] (see also [4]). In Subsection 6.1, a set of linear parts of generators for $\operatorname{Lift}\left(\varphi_{k}\right)$ is obtained by our method for any $k \geq 2$.

Example 3.3. Let $\psi_{n}:\left(\mathbb{K}^{n}, 0\right) \rightarrow\left(\mathbb{K}^{2 n-1}, 0\right)$ be given by

$$
\psi_{n}\left(v_{1}, \ldots, v_{n-1}, y\right)=\left(v_{1}, \ldots, v_{n-1}, y^{2}, v_{1} y, \ldots, v_{n-1} y\right) .
$$

Then, it is known that $f$ is an isolated stable mono-germ by [29] or [30 or [16. Thus, by Proposition $40 \bar{\omega} \psi_{n}$ is bijective. Therefore, it follows that $i_{1}\left(\psi_{n}\right)=i_{2}\left(\psi_{n}\right)=0$. By Theorem 1 Lemma 1.1 and Propositions 5, 6, the minimal number of generators for $\operatorname{Lift}\left(\psi_{n}\right)$ can be calculated as follows:

$$
\begin{aligned}
& (2 n-1) \cdot\left(\begin{array}{c}
2 n-1 \\
1
\end{array}\right)-\left(((2 n-1)-n) \cdot{ }_{1} \delta(\varphi)+{ }_{1} \gamma(\varphi)-{ }_{0} \gamma(\varphi)\right) \\
= & (2 n-1)^{2}-((n-1) \cdot n \cdot 2+n \cdot(2-1)-(2-1)) \\
= & 2 n^{2}-3 n+2 .
\end{aligned}
$$

In the case that $n=2, \psi_{2}$ equals $\varphi_{2}$ of Example 3.2 Thus, in this case, It has been verified in [4] and [9] that the minimal number of generators for $\operatorname{Lift}\left(\psi_{n}\right)$ is 
exactly 4 in the complex case and a set of generators has been obtained in 44 and [11. In Subsection 6.2, a set of generators for $\operatorname{Lift}\left(\psi_{n}\right)$ is obtained by our method for any $n \geq 2$.

Example 3.4. Examples 3.1, 3.2 and 3.3 can be generalized as follows. Let $f$ : $(\mathbb{K}, 0) \rightarrow\left(\mathbb{K}^{p}, 0\right)(p \geq 2)$ be an analytic map-germ such that $2 \leq \delta(f)<\infty$ and let $F:\left(\mathbb{K} \times \mathbb{K}^{c}, 0\right) \rightarrow\left(\mathbb{K}^{p} \times \mathbb{K}^{c}, 0\right)$ be a $\mathcal{K}$-miniversal unfolding of $f$, where $\mathcal{K}$-miniversal unfolding of $f$ is a map-germ given by (5.8) of [15] with $c=r$. Then, by [15 or [16, $F$ is an isolated stable mono-germ. Thus, by Proposition $4 .{ }_{0} \bar{\omega} F$ is bijective. Note that $c=p \delta(f)-1-p$ by theorem 4.5.1 of 28. By Theorem 11. Lemma 1.1 and Propositions 5, 6, the minimal number of generators for $\operatorname{Lift}(F)$ can be calculated as follows:

$$
\begin{aligned}
& (p+c) \cdot\left(\begin{array}{c}
p+c \\
1
\end{array}\right)-\left(((p+c)-(1+c)) \cdot{ }_{1} \delta(F)+{ }_{1} \gamma(F)-{ }_{0} \gamma(F)\right) \\
= & (p+c)^{2}-((p-1) \cdot(1+c) \cdot \delta(f)+c \cdot(\delta(f)-1)) \\
= & p^{2} \cdot \delta(f)-p \cdot \delta(f)+\delta(f)-p .
\end{aligned}
$$

By Mather's classification theorem (theorem A of [15]), proposition (1.6) of [15, Mather's normal form theorem for a stable map-germ (theorem (5.10) of [15]), the fact that the sharp inequality $p^{2} \delta(f)-p \delta(f)+\delta(f)-p>p+c$ holds (since $p, \delta(f) \geq 2$ ) and the fact that the module of liftable vector fields over an immersive stable multigerm is a free module if and only if $p=n+1$, we have the following:

Proposition 7. Let $f:\left(\mathbb{K}^{n}, S\right) \rightarrow\left(\mathbb{K}^{p}, 0\right)(n<p)$ be a stable multigerm of corank at most one. Then, Lift $(f)$ is a free module if and only if the properties $p=n+1$ and $\delta(f)=|S|$ are satisfied.

Example 3.5. Let $f:\left(\mathbb{K}^{2}, S\right) \rightarrow\left(\mathbb{K}^{2}, 0\right)$ be given by $(x, y) \mapsto\left(x, y^{2}\right),(x, y) \mapsto$ $\left(x^{2}, y\right)$. Then, it is known that $f$ is an isolated multigerm by 31] or [16]. Thus, by Proposition 4, $0 \bar{\omega} f$ is bijective. Therefore, it follows that $i_{1}(f)=i_{2}(f)=0$. By Theorem 1, Lemma 1.1 and Propositions [5, 6, the minimal number of generators for $\operatorname{Lift}(f)$ is the following:

$$
\begin{aligned}
& \operatorname{dim}_{\mathbb{K}} \frac{m_{0} \theta_{0}(2)}{m_{0}^{2} \theta_{0}(2)}-\left((2-2)_{1} \delta(f)+{ }_{1} \gamma(f)-{ }_{0} \gamma(f)\right) \\
= & 2^{2}-((2-2) \times 2 \times 4+2 \times(4-2)-(4-2)) \\
= & 2 .
\end{aligned}
$$

In this case we can construct easily a basis of $\operatorname{Lift}(f)$ consisting of 2 vector fields (see Subsection 6.4).

Example 3.6. Let $f:(\mathbb{K}, S) \rightarrow\left(\mathbb{K}^{2}, 0\right)$ be any one of the following three.

(1) $x \mapsto\left(x^{4}, x^{5}+x^{7}\right)$ (taken from [3]).

(2) $x \mapsto\left(x^{2}, x^{3}\right), x \mapsto\left(x^{3}, x^{2}\right)$ (taken from [12]).

(3) $x \mapsto(x, 0), x \mapsto(0, x), x \mapsto\left(x^{2}, x^{3}+x^{4}\right)$ (taken from [12]).

It has been shown in [3] or [12] that $T \mathcal{K}(f)=T \mathcal{A}(f)$ is satisfied. Thus, by Proposition $4,1 \bar{\omega} f$ is surjective. We can confirm easily that the following equality holds.

$$
2 \cdot\left(\begin{array}{c}
2 \\
1
\end{array}\right)=(2-1) \cdot{ }_{1} \delta(f)+{ }_{1} \gamma(f)-{ }_{0} \gamma(f) .
$$


Thus, ${ }_{1} \bar{\omega} f$ is injective by Proposition 5 Therefore, it follows that $i_{1}(f)=i_{2}(f)=1$. By Theorem 1, Lemma 1.1 and Propositions 5, 6, the minimal number of generators for $\operatorname{Lift}(f)$ can be calculated as follows:

$$
\begin{aligned}
& 2 \cdot\left(\begin{array}{c}
3 \\
2
\end{array}\right)-\left((2-1) \cdot{ }_{2} \delta(f)+{ }_{2} \gamma(f)-{ }_{1} \gamma(f)\right) \\
= & 2 \cdot 3-((2-1) \cdot 1 \cdot 4+(4-|S|)-(4-|S|)) \\
= & 2 .
\end{aligned}
$$

In the case $\mathbb{K}=\mathbb{C}$, it has been known that any plane algebraic curve is a free divisor by [27]. Thus, by combining [6] and [27, it has been known that the minimal number of generators for $\operatorname{Lift}(f)$ is 2 in the complex case.

Example 3.7. Let $f:\left(\mathbb{K}^{2}, 0\right) \rightarrow\left(\mathbb{K}^{2}, 0\right)$ be given by $f(x, y)=\left(x, x y+y^{5} \pm y^{7}\right)$ (taken from 24]). It has been shown in 24 that $T \mathcal{K}(f)=T \mathcal{A}(f)$ is satisfied. Thus, by Proposition $4, \bar{\omega} f$ is surjective. It is easily seen that the following equality holds.

$$
2 \cdot\left(\begin{array}{c}
2 \\
1
\end{array}\right)=(2-2) \cdot{ }_{1} \delta(f)+{ }_{1} \gamma(f)-{ }_{0} \gamma(f) .
$$

Thus, ${ }_{1} \bar{\omega} f$ is injective by Proposition 5 . Therefore, it follows that $i_{1}(f)=i_{2}(f)=1$. By Theorem 1, Lemma 1.1 and Propositions 5, 6, the minimal number of generators for Lift $(f)$ can be calculated as follows.

$$
\begin{aligned}
& 2 \cdot\left(\begin{array}{c}
3 \\
2
\end{array}\right)-\left((2-2) \cdot{ }_{2} \delta(f)+{ }_{2} \gamma(f)-{ }_{1} \gamma(f)\right) \\
= & 2 \cdot 3-((2-2) \cdot 3 \cdot 5+3 \cdot(5-1)-2 \cdot(5-1)) \\
= & 2 .
\end{aligned}
$$

As same as Example 3.6 it has been known that the minimal number of generators for $\operatorname{Lift}(f)$ is 2 in the complex case.

Example 3.8. Let $f:\left(\mathbb{K}^{4}, 0\right) \rightarrow\left(\mathbb{K}^{5}, 0\right)$ be given by the following:

$$
f\left(x_{1}, x_{2}, x_{3}, y\right)=\left(x_{1}, x_{2}, x_{3}, y^{4}+x_{1} y, y^{6}+y^{7}+x_{2} y+x_{3} y^{2}\right) .
$$

This example is taken from [26] where the property $T \mathcal{K}(f)=T \mathcal{A}(f)$ has been shown. Thus, by Proposition $4{ }_{1} \bar{\omega} f$ is surjective. It is easily seen that the following equality holds.

$$
5 \cdot\left(\begin{array}{c}
5 \\
1
\end{array}\right)=(5-4) \cdot{ }_{1} \delta(f)+{ }_{1} \gamma(f)-{ }_{0} \gamma(f)
$$

Thus, $1 \bar{\omega} f$ is injective by Proposition 5 . Therefore, it follows that $i_{1}(f)=i_{2}(f)=1$. By Theorem 1. Lemma 1.1 and Propositions 5, 6, the minimal number of generators for Lift $(f)$ can be calculated as follows:

$$
\begin{aligned}
& 5 \cdot\left(\begin{array}{c}
6 \\
2
\end{array}\right)-\left((5-4) \cdot{ }_{2} \delta(f)+{ }_{2} \gamma(f)-{ }_{1} \gamma(f)\right) \\
= & 5 \cdot 15-((5-4) \cdot 10 \cdot 4+10 \cdot(4-1)-4 \cdot(4-1)) \\
= & 17 .
\end{aligned}
$$

Example 3.9. Let $c: \mathbb{K} \rightarrow \mathbb{K}^{2}$ be the map defined by $c(x)=\left(x^{2}, x^{3}\right)$. For any real number $\theta$, we let $R_{\theta}: \mathbb{K}^{2} \rightarrow \mathbb{K}^{2}$ be the rotation of $\mathbb{K}^{2}$ about the origin with 
respect to the angle $\theta$ :

$$
R_{\theta}\left(\begin{array}{c}
X \\
Y
\end{array}\right)=\left(\begin{array}{rr}
\cos \theta & -\sin \theta \\
\sin \theta & \cos \theta
\end{array}\right)\left(\begin{array}{l}
X \\
Y
\end{array}\right) .
$$

For any $i \in \mathbb{N}$, let $\theta_{0}, \ldots, \theta_{i}$ be real numbers such that $0 \leq \theta_{j}<2 \pi(0 \leq j \leq i)$ and $0 \neq\left|\theta_{j}-\theta_{k}\right| \neq \pi(j \neq k)$. Set $S=\left\{s_{0}, \ldots, s_{i}\right\}\left(s_{j} \neq s_{k}\right.$ if $\left.j \neq k\right)$ and define $c_{\theta_{j}}:\left(\mathbb{K}, s_{j}\right) \rightarrow\left(\mathbb{K}^{2}, 0\right)$ as $c_{\theta_{j}}(x)=R_{\theta_{j}} \circ c\left(x_{j}\right)$, where $x_{j}=x-s_{j}$. A multigerm $\left\{c_{\theta_{0}}, \ldots, c_{\theta_{i}}\right\}:(\mathbb{K}, S) \rightarrow\left(\mathbb{K}^{2}, 0\right)$, which is called a multicusp, is denoted by $c_{\left(\theta_{0}, \ldots, \theta_{i}\right)}$.

In [18, it has been shown that $i_{1}\left(c_{\left(\theta_{0}, \ldots, \theta_{i}\right)}\right)=i_{2}\left(c_{\left(\theta_{0}, \ldots, \theta_{i}\right)}\right)=i$ for any $i \in \mathbb{N}$. Thus, by Theorem 1. Lemma 1.1 and Propositions 5 [ 6. the minimal number of generators for $\operatorname{Lift}\left(c_{\left(\theta_{0}, \ldots, \theta_{i}\right)}\right)$ can be calculated as follows.

$$
\begin{aligned}
& 2 \cdot\left(\begin{array}{c}
2+i \\
i+1
\end{array}\right)-\left((2-1) \cdot{ }_{i+1} \delta\left(c_{\left(\theta_{0}, \ldots, \theta_{i}\right)}\right)+{ }_{i+1} \gamma\left(c_{\left(\theta_{0}, \ldots, \theta_{i}\right)}\right)-{ }_{i} \gamma\left(c_{\left(\theta_{0}, \ldots, \theta_{i}\right)}\right)\right) \\
= & 2 \cdot(2+i)-\left((2-1) \cdot 1 \cdot \delta\left(c_{\left(\theta_{0}, \ldots, \theta_{i}\right)}\right)+1 \cdot \gamma\left(c_{\left(\theta_{0}, \ldots, \theta_{i}\right)}\right)-1 \cdot \gamma\left(c_{\left(\theta_{0}, \ldots, \theta_{i}\right)}\right)\right) \\
= & 2 .
\end{aligned}
$$

As same as Example 3.6. it has been already known that the minimal number of generators for $\operatorname{Lift}\left(c_{\left(\theta_{0}, \ldots, \theta_{i}\right)}\right)$ is 2 in the complex case.

\section{Proof of Theorem 1}

Since ${ }_{i} \bar{\omega} f$ is surjective, by Lemma 1.1 we have that ${ }_{j} \bar{\omega} f$ is surjective for any $j>i$. Since ${ }_{i} \bar{\omega} f$ is injective, any $\eta \in \theta_{0}(p)$ such that $\omega f(\eta) \in T \mathcal{R}_{e}(f)$ is contained in $m_{0}^{i+1} \theta_{0}(p)$. Set $\rho(f)=\operatorname{dim}_{\mathbb{K}} \operatorname{ker}\left({ }_{i+1} \bar{\omega} f\right)$. Then, since ${ }_{i} \bar{\omega} f$ is bijective, $\rho(f)$ must be positive by Corollary 1 . Let $\left\{\eta_{1}+m_{0}^{i+2} \theta_{0}(p), \ldots, \eta_{\rho(f)}+m_{0}^{i+2} \theta_{0}(p)\right\}$ be a basis of $\operatorname{ker}(i+1 \bar{\omega} f)$. Then, we have that

$$
\eta_{j} \circ f \in T \mathcal{R}_{e}(f) \cap f^{*} m_{0}^{i+1} \theta_{S}(f)+f^{*} m_{0}^{i+2} \theta_{S}(f) \quad(1 \leq j \leq \rho(f)) .
$$

Since ${ }_{i+2} \bar{\omega} f$ is surjective, we have the following:

$$
\begin{aligned}
& T \mathcal{R}_{e}(f) \cap f^{*} m_{0}^{i+1} \theta_{S}(f)+f^{*} m_{0}^{i+2} \theta_{S}(f) \\
= & T \mathcal{R}_{e}(f) \cap f^{*} m_{0}^{i+1} \theta_{S}(f)+T \mathcal{R}_{e}(f) \cap f^{*} m_{0}^{i+2} \theta_{S}(f)+\omega f\left(m_{0}^{i+2} \theta_{0}(p)\right) .
\end{aligned}
$$

Thus, for any $j(1 \leq j \leq \rho(f))$ there exists $\widetilde{\eta}_{j} \in m_{0}^{i+2} \theta_{0}(p)$ such that $\left(\eta_{j}+\widetilde{\eta}_{j}\right) \circ f \in$ $T \mathcal{R}_{e}(f) \cap T \mathcal{L}_{e}(f)$. Let $A$ be the $C_{0}$-module generated by $\eta_{j}+\widetilde{\eta}_{j}(1 \leq j \leq \rho(f))$.

Let $\hat{\omega} f: \theta_{0}(p) \rightarrow \frac{\theta_{S}(f)}{T \mathcal{R}_{e}(f)}$ be given by $\hat{\omega} f(\eta)=\omega f(\eta)+T \mathcal{R}_{e}(f)$. Then, $\operatorname{ker}(\hat{\omega} f)$ is the set of vector fields liftable over $f$. In order to show that $\operatorname{ker}(\hat{\omega} f)=A$, we consider the following commutative diagram.

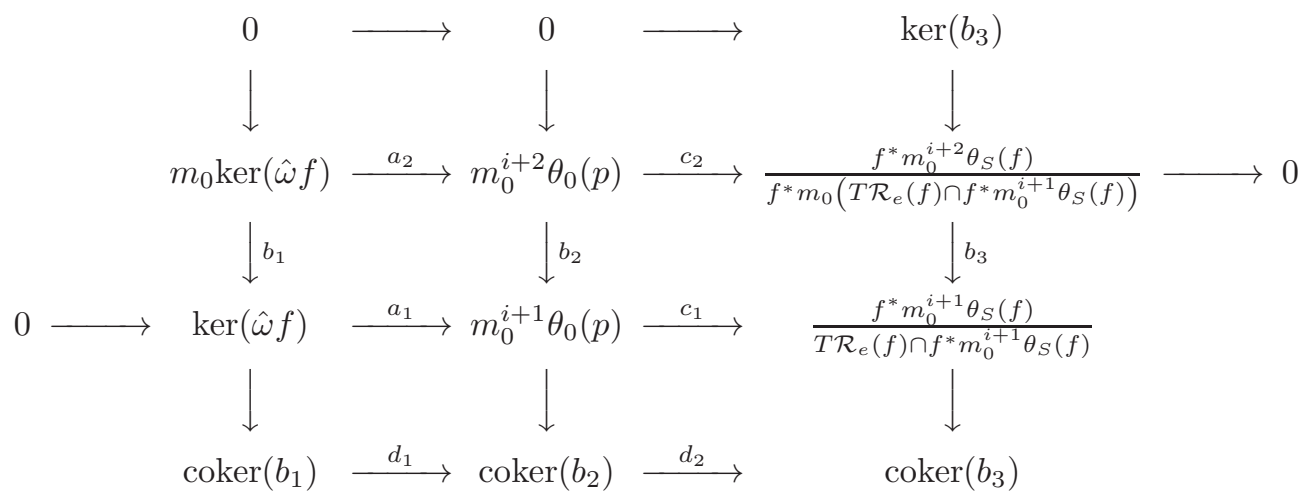


Here, $a_{j}(j=1,2), b_{j}(j=1,2)$ are inclusions, $b_{3}$ is defined by $b_{3}\left([\eta]_{i+2}\right)=[\eta]_{i+1}$ and $c_{j}(j=1,2)$ are defined by $c_{j}(\eta)=[\omega f(\eta)]_{i+j}$, where $[\eta]_{i+1}=\eta+T \mathcal{R}_{e}(f) \cap$ $f^{*} m_{0}^{i+1} \theta_{S}(f)$ and $[\eta]_{i+2}=\eta+f^{*} m_{0}\left(T \mathcal{R}_{e}(f) \cap f^{*} m_{0}^{i+1} \theta_{S}(f)\right)$.

\section{Lemma 4.1.}

$$
f^{*} m_{0}\left(T \mathcal{R}_{e}(f) \cap f^{*} m_{0}^{i+1} \theta_{S}(f)\right)=T \mathcal{R}_{e}(f) \cap f^{*} m_{0}^{i+2} \theta_{S}(f) .
$$

Proof of Lemma 4.1. It is clear that $f^{*} m_{0}\left(T \mathcal{R}_{e}(f) \cap f^{*} m_{0}^{i+1} \theta_{S}(f)\right) \subset T \mathcal{R}_{e}(f) \cap$

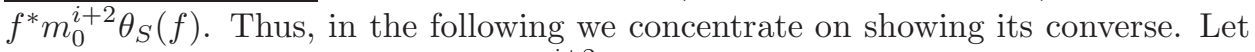
$\eta$ be an element of $T \mathcal{R}_{e}(f) \cap f^{*} m_{0}^{i+2} \theta_{S}(f)$. Let $f_{j}$ be a branch of $f$, namely, $f_{j}=\left.f\right|_{\left(\mathbb{K}^{n}, s_{j}\right)}(1 \leq j \leq|S|)$. Then, we have that $\eta \in T \mathcal{R}_{e}\left(f_{j}\right) \cap f_{j}^{*} m_{0}^{i+2} \theta_{s_{j}}\left(f_{j}\right)$ for any $j(1 \leq j \leq|S|)$. Thus, there exists $\xi_{j} \in \theta_{s_{j}}(n)$ such that $t f_{j}\left(\xi_{j}\right)=\eta$.

Since $f$ is of corank at most one, for any $j(1 \leq j \leq|S|)$ there exist germs of diffeomorphism $h_{j}:\left(\mathbb{K}^{n}, s_{j}\right) \rightarrow\left(\mathbb{K}^{n}, s_{j}\right)$ and $H_{j}:\left(\mathbb{K}^{p}, 0\right) \rightarrow\left(\mathbb{K}^{p}, 0\right)$ such that $H_{j} \circ f_{j} \circ h_{j}^{-1}$ has the following form:

$$
\begin{aligned}
& H_{j} \circ f_{j} \circ h_{j}^{-1}(x, y) \\
= & \left(x, y^{\delta\left(f_{j}\right)}+f_{j, n}(x, y), f_{j, n+1}(x, y), \ldots, f_{j, p}(x, y)\right) .
\end{aligned}
$$

Here, $x$ stands for $\left(x_{1}, \ldots, x_{n-1}\right)$ and $x_{1}, \ldots, x_{n-1}, y$ are local coordinates of the coordinate system $\left(U_{j}, h_{j}\right)$ at $s_{j}$ and $f_{j, q}$ satisfies $f_{j, q}(0, \ldots, 0, y)=o\left(y^{\delta\left(f_{j}\right)}\right)$ for any $q(n \leq q \leq p)$. Set

$$
\xi_{j}=\sum_{m=1}^{n-1} \xi_{j, m} \frac{\partial}{\partial x_{m}}+\xi_{j, n} \frac{\partial}{\partial y} \text { and } \eta=\sum_{q=1}^{p} \eta_{q} \frac{\partial}{\partial X_{q}} .
$$

Then, by the above form of $H_{j} \circ f_{j} \circ h_{j}^{-1}$ and the equality $t f_{j}\left(\xi_{j}\right)=\eta$, the following hold:

$$
\begin{aligned}
& \xi_{j, m}\left(x_{1}, \ldots, x_{n-1}, y\right)=\eta_{m}\left(x_{1}, \ldots, x_{n-1}, y\right) \quad(1 \leq m \leq n-1) \\
& \lambda\left(x_{1}, \ldots, x_{n-1}, y\right) \xi_{j, n}\left(x_{1}, \ldots, x_{n-1}, y\right)=\mu\left(x_{1}, \ldots, x_{n-1}, y\right),
\end{aligned}
$$

where $\lambda=\delta\left(f_{j}\right) y^{\delta\left(f_{j}\right)-1}+\frac{\partial f_{j, n}}{\partial y}$ and $\mu=\eta_{n}-\sum_{m=1}^{n-1} \xi_{j, m} \frac{\partial f_{j, n}}{\partial x_{m}}$. Since $\eta_{q} \in f^{*} m_{0}^{i+2} C_{s_{j}}$ for any $q(1 \leq q \leq p)$, by (4.1) we have that $\xi_{j, m} \in f^{*} m_{0}^{i+2} C_{s_{j}}$ for any $m(1 \leq m \leq$ $n-1)$.

Since $f_{j, q}(0, \ldots, 0, y)=o\left(y^{\delta\left(f_{j}\right)}\right)$ for any $q(n \leq q \leq p)$, we have the following properties:

(1) $Q\left(f_{j}\right)=Q\left(x_{1}, \ldots, x_{n-1}, y^{\delta\left(f_{j}\right)}\right)=Q\left(x_{1}, \ldots, x_{n}, y^{\lambda}\right)$.

(2) $[1],[y], \ldots,\left[y^{\delta\left(f_{j}\right)-2}\right],[\lambda]$ constitute a basis of $Q\left(f_{j}\right)$.

Thus, by the preparation theorem, $C_{s_{j}}$ is generated by $1, y, \ldots, y^{\delta\left(f_{j}\right)-2}, \lambda$ as $C_{0^{-}}$ module via $f_{j}$. Therefore, for any positive integer $r, f_{j}^{*} m_{0}^{r} C_{s_{j}}$ is generated by 
elements of the union of the following three sets $U_{r}, V_{r}, W_{r}$ as $C_{0}$-module via $f_{j}$.

$$
\begin{aligned}
U_{r} & =\left\{x_{1}^{k_{1}} \cdots x_{n-1}^{k_{n-1}} \lambda^{k_{n}} y^{k_{n}+\ell} \mid k_{m} \geq 0, \sum_{m=1}^{n-1} k_{m}=r-k_{n}<r, 0 \leq \ell \leq \delta\left(f_{j}\right)-2\right\}, \\
V_{r} & =\left\{x_{1}^{k_{1}} \cdots x_{n-1}^{k_{n-1}} \lambda^{k_{n}+1} y^{k_{n}} \mid k_{m} \geq 0, \sum_{m=1}^{n-1} k_{m}=r-k_{n},\right\}, \\
W_{r} & =\left\{x_{1}^{k_{1}} \cdots x_{n-1}^{k_{n-1}} y^{\ell} \mid k_{m} \geq 0, \sum_{m=1}^{n-2} k_{m}=r, 0 \leq \ell \leq \delta\left(f_{j}\right)-2\right\} .
\end{aligned}
$$

Then, by using these notations, for any $m(1 \leq m \leq n-1) \xi_{j, m}$ can be expressed as follows:

$$
\xi_{j, m}=\sum_{u \in U_{i+2}} \varphi_{u, j, m} u+\sum_{v \in V_{i+2}} \varphi_{v, j, m} v+\sum_{w \in W_{i+2}} \varphi_{w, j, m} w
$$

where $\varphi_{u, j, m}, \varphi_{v, j, m}, \varphi_{w, j, m}$ are some elements of $C_{s_{j}}$.

Next, we investigate $\xi_{j, n}$. Since $\mu$ has the form $\mu=\eta_{n}-\sum_{m=1}^{n-1} \xi_{j, m} \frac{\partial f_{j, n}}{\partial x_{m}}$ and $\eta_{n}, \xi_{j, m}$ are contained in $f^{*} m_{0}^{i+2} C_{s_{j}}, \mu$ is contained in $f^{*} m_{0}^{i+2} C_{s_{j}}$. On the other hand, $\lambda$ must divide $\mu$ by (4.2). Thus, $\mu$ is generated by elements of $U_{i+2} \cup V_{i+2}$. Hence, $\xi_{j, n}=\frac{\mu}{\lambda}$ can be expressed as follows:

$$
\xi_{j, n}=\sum_{u \in U_{i+2}} \varphi_{u, j, n} \frac{u}{\lambda}+\sum_{v \in V_{i+2}} \varphi_{v, j, n} \frac{v}{\lambda}
$$

where $\varphi_{u, j, n}, \varphi_{v, j, n}$ are some elements of $C_{s_{j}}$. Since $\frac{u}{\lambda} \in U_{i+1} \cup V_{i+1} \cup W_{i+1}$ for any $u \in U_{i+2}$ and $\frac{v}{\lambda} \in U_{i+1}$ (resp., $\left.\frac{v}{\lambda} \in V_{i+1}\right)$ if $\delta\left(f_{j}\right) \geq 2$ (resp., $\delta\left(f_{j}\right)=1$ ) for any $v \in V_{i+2}, \xi_{j, n}$ is belonging to $f_{j}^{*} m_{0}^{i+1} C_{s_{j}}$.

Since $f_{j}^{*} m_{0}^{i+2} C_{s_{j}} \subset f_{j}^{*} m_{0}^{i+1} C_{s_{j}}$, for any $j(1 \leq j \leq|S|)$ and any $m(1 \leq m \leq$ $n-1)$, we have the following:

$$
\begin{aligned}
& t f_{j}\left(\xi_{j, m} \frac{\partial}{\partial x_{m}}\right) \\
=t f_{j} & \left.\left(\sum_{u \in U_{i+1}} \widetilde{\varphi}_{u, j, m} u+\sum_{v \in V_{i+1}} \widetilde{\varphi}_{v, j, m} v+\sum_{w \in W_{i+1}} \widetilde{\varphi}_{w, j, m} w\right) \frac{\partial}{\partial x_{m}}\right) \\
= & \sum_{u \in U_{i+1}} u\left(t f_{j}\left(\widetilde{\varphi}_{u, j, m} \frac{\partial}{\partial x_{m}}\right)\right)+\sum_{v \in V_{i+1}} v\left(t f_{j}\left(\widetilde{\varphi}_{v, j, m} \frac{\partial}{\partial x_{m}}\right)\right) \\
& +\sum_{w \in W_{i+1}} w\left(t f_{j}\left(\widetilde{\varphi}_{w, j, m} \frac{\partial}{\partial x_{m}}\right)\right),
\end{aligned}
$$


where $\widetilde{\varphi}_{u, j, m}, \widetilde{\varphi}_{v, j, m}, \widetilde{\varphi}_{w, j, m}$ are some elements of $C_{s_{j}}$. Moreover, for any $j(1 \leq j \leq$ $|S|)$ we have the following:

$$
\begin{gathered}
t f_{j}\left(\xi_{j, n} \frac{\partial}{\partial y}\right) \\
=t f_{j}\left(\left(\sum_{u \in U_{i+1}} \psi_{u, j, n} u+\sum_{v \in V_{i+1}} \psi_{v, j, n} v+\sum_{w \in W_{i+1}} \psi_{w, j, n} w\right) \frac{\partial}{\partial y}\right) \\
=\sum_{u \in U_{i+1}} u\left(t f_{j}\left(\psi_{u, j, n} \frac{\partial}{\partial y}\right)\right)+\sum_{v \in V_{i+1}} v\left(t f_{j}\left(\psi_{v, j, n} \frac{\partial}{\partial y}\right)\right) \\
+\sum_{w \in W_{i+1}} w\left(t f_{j}\left(\psi_{w, j, n} \frac{\partial}{\partial y}\right)\right)
\end{gathered}
$$

where $\psi_{u, j, n}, \psi_{v, j, n}, \psi_{w, j, n}$ are elements of $C_{s_{j}}$. Since the union $U_{i+1} \cup V_{i+1} \cup W_{i+1}$ is a finite set, we have the following:

$$
\eta=t f_{j}\left(\sum_{m=1}^{n-1} \xi_{j, m} \frac{\partial}{\partial x_{m}}+\xi_{j, n} \frac{\partial}{\partial y}\right) \in f_{j}^{*} m_{0}^{i+1}\left(T \mathcal{R}_{e}\left(f_{j}\right) \cap f_{j}^{*} m_{0} \theta_{s_{j}}\left(f_{j}\right)\right) .
$$

Notice that $i+1 \geq 1$ and $f_{j}$ is any branch of $f$. Hence, we have that

$$
\eta \in f^{*} m_{0}\left(T \mathcal{R}_{e}(f) \cap f^{*} m_{0}^{i+1} \theta_{S}(f)\right) .
$$

Lemma 4.1 implies that $c_{2}$ is surjective, thus even the second row sequence is exact. Lemma 4.1 implies also that $b_{3}$ is injective and thus $\operatorname{ker}\left(b_{3}\right)=0$. Hence, by the snake lemma, we see that $d_{1}$ is injective. On the other hand, since there exists an isomorphism

$$
\varphi: \frac{f^{*} m_{0}^{i+1} \theta_{S}(f)}{T \mathcal{R}_{e}(f) \cap f^{*} m_{0}^{i+1} \theta_{S}(f)+f^{*} m_{0}^{i+2} \theta_{S}(f)} \rightarrow \operatorname{coker}\left(b_{3}\right)
$$

such that $d_{2}=\varphi \circ_{i+1} \bar{\omega} f$ we have that $\operatorname{ker}\left(d_{2}\right)=\operatorname{ker}\left(\varphi \circ{ }_{i+1} \bar{\omega} f\right)=\operatorname{ker}\left({ }_{i+1} \bar{\omega} f\right)$. Therefore, we have the following:

$$
\operatorname{dim}_{\mathbb{K}} \frac{\operatorname{ker}(\hat{\omega} f)}{m_{0} \operatorname{ker}(\hat{\omega} f)}=\operatorname{dim}_{\mathbb{K}} \operatorname{ker}(i+1 \bar{\omega} f)=\rho(f)=\operatorname{dim}_{\mathbb{K}} \frac{A}{m_{0} A} .
$$

Moreover, $A$ is a submodule of $\operatorname{ker}(\hat{\omega} f)$ and our category is the analytic category. Therefore, we have that $\operatorname{ker}(\hat{\omega} f)=A$.

Q.E.D.

\section{Proof of Theorem 2}

We first show the assertion (1) of Theorem 2, Let $F(x, \lambda)=\left(f_{\lambda}(x), \lambda\right)\left(f_{0}=f\right)$ be a stable unfolding of $f$, Since $\eta$ is an element of $\operatorname{Lift}(F)$, by definition, there exists a vector field $\xi \in \theta_{S}(n+r)$ such that $d F \circ \xi=\eta \circ F$. Set $x=\left(x_{1}, \ldots, x_{n}\right), \lambda=$ $\left(\lambda_{1}, \ldots, \lambda_{r}\right), f_{\lambda}=\left(f_{\lambda, 1}, \ldots, f_{\lambda, p}\right)$ and $\xi(x, \lambda)=\left(\xi_{1}(x, \lambda), \ldots, \xi_{n+r}(x, \lambda)\right)$. Then, we 
have the following:

$$
\left(\begin{array}{cc}
\frac{\partial f_{\lambda}}{\partial x}(x, \lambda) & \frac{\partial f_{\lambda}}{\lambda}(x, \lambda) \\
0 & E_{r}
\end{array}\right)\left(\begin{array}{c}
\xi_{1}(x, \lambda) \\
\vdots \\
\xi_{n}(x, \lambda) \\
\xi_{n+1}(x, \lambda) \\
\vdots \\
\xi_{n+r}(x, \lambda)
\end{array}\right)=\left(\begin{array}{c}
\eta_{1}(F(x, \lambda)) \\
\vdots \\
\eta_{p}(F(x, \lambda)) \\
\eta_{p+1}(F(x, \lambda)) \\
\vdots \\
\eta_{p+r}(F(x, \lambda))
\end{array}\right) .
$$

Here, $\frac{\partial f_{\lambda}}{\partial x}(x, \lambda)$ is the $p \times n$ matrix whose $(i, j)$ elements is $\frac{\partial f_{\lambda, i}}{\partial x_{j}}(x, \lambda), \frac{\partial f_{\lambda}}{\partial \lambda}(x, \lambda)$ is the $p \times r$ matrix whose $(i, k)$ elements is $\frac{\partial f_{\lambda, i}}{\partial \lambda_{k}}(x, \lambda)$, and $E_{r}$ stands for the $r \times r$ unit matrix. In particular, we have the following two:

$$
\left(\frac{\partial f_{\lambda}}{\partial x}(x, 0)\right)\left(\begin{array}{c}
\xi_{1}(x, 0) \\
\vdots \\
\xi_{n}(x, 0)
\end{array}\right)+\left(\frac{\partial f_{\lambda}}{\partial \lambda}(x, 0)\right)\left(\begin{array}{c}
\xi_{n+1}(x, 0) \\
\vdots \\
\xi_{n+r}(x, 0)
\end{array}\right)=\left(\begin{array}{c}
\eta_{1}(F(x, 0)) \\
\vdots \\
\eta_{p}(F(x, 0))
\end{array}\right)
$$

and

$$
\xi_{n+k}(x, 0)=\eta_{p+k}(F(x, 0))=\eta_{p+k}(f(x), 0)=0 \quad(1 \leq k \leq r) .
$$

The last equality of $(*)$ is obtained from the assumption that $\eta \in \operatorname{Lift}(g)$ and the fact that $\operatorname{Lift}(g)$ is generated by $\frac{\partial}{\partial X_{1}}, \ldots, \frac{\partial}{\partial X_{p}}$ and $\Lambda_{1} \frac{\partial}{\partial \Lambda_{1}}, \ldots, \Lambda_{r} \frac{\partial}{\partial \Lambda_{r}}$. Thus, we have the following:

$$
\left(\frac{\partial f_{\lambda}}{\partial x}(x, 0)\right)\left(\begin{array}{c}
\xi_{1}(x, 0) \\
\vdots \\
\xi_{n}(x, 0)
\end{array}\right)=\left(\begin{array}{c}
\eta_{1}(F(x, 0)) \\
\vdots \\
\eta_{p}(F(x, 0))
\end{array}\right) .
$$

Therefore, $\bar{\eta}$ is a liftable vector field of $f$.

We next show the assertion (2) of Theorem 2 Since $\bar{\eta} \in \operatorname{Lift}(f)$, by definition, there exists a vector field $\bar{\xi} \in \theta_{S}(n)$ such that $d f(\bar{\xi})=\bar{\eta} \circ f$. Set $\eta(X, \Lambda)=(\bar{\eta}(X), 0)$ and $\xi(x, \lambda)=(\bar{\xi}(x), 0)$. Set also $\widetilde{\eta}=\eta \circ F-d F(\xi) \in \theta_{S \times\{0\}}(F)$. It is not difficult to see that $\widetilde{\eta}(x, 0)=(0,0)$. Thus, by the preparation theorem, there must exist a vector field $\widetilde{\eta}_{1} \in \theta_{S \times\{0\}}(F)$ such that $\widetilde{\eta}=\Lambda \widetilde{\eta}_{1}$.

Since $F$ is stable, there exist $\hat{\xi} \in \theta_{S \times\{0\}}(n+1)$ and $\hat{\eta} \in \theta_{(0,0)}(p+1)$ such that $\widetilde{\eta}_{1}=d F(\hat{\xi})+\hat{\eta} \circ F$. We therefore have that

$$
\eta \circ F-d F(\xi)=\widetilde{\eta}=\Lambda \widetilde{\eta}_{1}=\lambda(d F(\hat{\xi})+\hat{\eta} \circ F)=d F(\lambda \hat{\xi})+(\Lambda \hat{\eta}) \circ F .
$$

It is clear that $\eta-\Lambda \hat{\eta} \in \operatorname{Lift}(F)$. Moreover, we have that the $(p+1)$ component of $\eta-\Lambda \hat{\eta}$ is $0-(\Lambda \hat{\eta})_{p+1}=-\Lambda(\hat{\eta})_{p+1}$, which implies that $\eta-\Lambda \widetilde{\eta} \in \operatorname{Lift}\left(g_{1}\right)$. Q.E.D.

\section{How to CONSTRUCT LIFTABLE VECTOR FIELDS}

In principle, the proof of Theorem 1 provides how to construct generators for the module of liftable vector fields over a given finitely determined multigerm $f$ satisfying the assumption of Theorem 1. In Subsections 6.1 6.5. we examine it by several examples. In Subsections 6.6] and 6.7 as an application of Thereom 2 we explain how to construct all liftable vector fields over a given analytic multigerm admitting a one-parameter stable unfolding by several examples. 
6.1. $\operatorname{Lift}\left(\varphi_{k}\right)$ for $\varphi_{k}\left(u_{1}, \ldots, u_{k-2}, v_{1}, \ldots, v_{k-1}, y\right)=$ $\left(u_{1}, \ldots, u_{k-2}, v_{1}, \ldots, v_{k-1}, y^{k}+\sum_{i=1}^{k-2} u_{i} y^{i}, \sum_{i=1}^{k-1} v_{i} y^{i}\right)$.

Since the purpose of this subsection is to examine that the proof of Theorem 1 in principle provides how to construct generators for the module of liftable vector fields, in order to avoid just long calculations, in this subsection we restrict ourselves to obtain only the linear parts of generators for the module of liftable vector fields over $\varphi_{k}$ of Example 3.2 Note that the linear parts of generators themselves are already useful to obtain the best lower bound for the $\mathcal{A}_{e}$ codimensions of some multigerms (see [22]).

Definition 6.1. Let $\eta_{1}, \eta_{2}$ be vector fields along $\varphi_{k}$ (namely, $\left.\eta_{1}, \eta_{2} \in \theta_{S}\left(\varphi_{k}\right)\right)$.

(1) We denote $\left.\eta_{1} \equiv \eta_{2}\left(\bmod T \mathcal{R}_{e}\left(\varphi_{k}\right)\right)\right)$ if $\eta_{1}-\eta_{2} \in T \mathcal{R}_{e}\left(\varphi_{k}\right)$.

(2) We denote $\left.\eta_{1} \equiv \eta_{2}\left(\bmod T \mathcal{R}_{e}\left(\varphi_{k}\right)+\varphi_{k}^{*} m_{0}^{2} \theta_{S}\left(\varphi_{k}\right)\right)\right)$ if $\eta_{1}-\eta_{2} \in T \mathcal{R}_{e}\left(\varphi_{k}\right)+$ $\varphi_{k}^{*} m_{0}^{2} \theta_{S}\left(\varphi_{k}\right)$.

Let $\left(U_{1}, \ldots, U_{k-2}, V_{1}, \ldots, V_{k-1}, W_{1}, W_{2}\right)$ be the standard coordinates of $\mathbb{K}^{2 k-1}$. Along the proof of Theorem 1, we first look for clues of linear terms of liftable vector fields in $\frac{\varphi_{k}^{*} m_{0} \theta_{S}\left(\varphi_{k}\right)}{\varphi_{k}^{*} m_{0}^{2} \theta_{S}\left(\varphi_{k}\right)}$. From the form of the Jacobian matrix of $\varphi_{k}$ and since the minimal number of generators is $(3 k-2)$ by Example 3.2 we can guess that clues of linear terms of liftable vector fields are the following $(3 k-2)$ vector fields along $\varphi_{k}$ :

$$
\begin{aligned}
& \left(W_{1} \circ \varphi_{k}\right) \frac{\partial}{\partial W_{1}},\left(W_{2} \circ \varphi_{k}\right) \frac{\partial}{\partial W_{1}},\left(W_{2} \circ \varphi_{k}\right) \frac{\partial}{\partial W_{2}}, \\
& y^{i}\left(W_{1} \circ \varphi_{k}\right) \frac{\partial}{\partial W_{1}} \quad(1 \leq i \leq k-2), \\
& y^{i}\left(W_{2} \circ \varphi_{k}\right) \frac{\partial}{\partial W_{1}} \quad(1 \leq i \leq k-2), \\
& y^{i}\left(W_{2} \circ \varphi_{k}\right) \frac{\partial}{\partial W_{2}} \quad(1 \leq i \leq k-1) .
\end{aligned}
$$

First we try to find a vector field $\eta \in m_{0} \theta_{0}(2 k-1)$ such that $W_{1} \frac{\partial}{\partial W_{1}} \neq \eta$ and $\left(W_{1} \circ \varphi_{k}\right) \frac{\partial}{\partial W_{1}} \equiv \eta \circ \varphi_{k}\left(\bmod T \mathcal{R}_{e}\left(\varphi_{k}\right)\right)$ because $W_{1} \frac{\partial}{\partial W_{1}}-\eta$ must be a liftable vector field for such a $\eta$.

$$
\begin{aligned}
& \left(W_{1} \circ \varphi_{k}\right) \frac{\partial}{\partial W_{1}} \\
\equiv & \left(y^{k}+\sum_{i=1}^{k-2} u_{i} y^{i}\right) \frac{\partial}{\partial W_{1}} \\
\equiv & \left(-\frac{1}{k} \sum_{i=1}^{k-2} i u_{i} y^{i}+\sum_{i=1}^{k-2} u_{i} y^{i}\right) \frac{\partial}{\partial W_{1}}-\sum_{i=1}^{k-1} i v_{i} y^{i-1} \frac{\partial}{\partial W_{2}} \quad\left(\bmod T \mathcal{R}_{e}\left(\varphi_{k}\right)\right) \\
\equiv & -\sum_{i=1}^{k-2} \frac{k-i}{k}\left(U_{i} \circ \varphi_{k}\right) \frac{\partial}{\partial U_{i}}+\sum_{i=2}^{k-1} i\left(V_{i} \circ \varphi_{k}\right) \frac{\partial}{\partial V_{i-1}} \\
& +\left(V_{1} \circ \varphi_{k}\right) \frac{\partial}{\partial W_{2}} \quad\left(\bmod T \mathcal{R}_{e}\left(\varphi_{k}\right)\right) .
\end{aligned}
$$


Thus, it follows that the following is a liftable vector fields over $\varphi_{k}$ where $\widetilde{\eta}_{1}=0$.

$$
\eta_{1}+\widetilde{\eta}_{1}=\sum_{i=1}^{k-2} \frac{k-i}{k} U_{i} \frac{\partial}{\partial U_{i}}-\sum_{i=2}^{k-1} i V_{i} \frac{\partial}{\partial V_{i-1}}+W_{1} \frac{\partial}{\partial W_{1}}-V_{1} \frac{\partial}{\partial W_{2}} .
$$

Secondly, we try to find a vector field $\eta \in m_{0} \theta_{0}(2 k-1)$ such that $W_{2} \frac{\partial}{\partial W_{1}} \neq \eta$ and $\left.\left(W_{2} \circ \varphi_{k}\right) \frac{\partial}{\partial W_{1}} \equiv \eta \circ \varphi_{k}\left(\bmod T \mathcal{R}_{e}\left(\varphi_{k}\right)\right)+\varphi_{k}^{2} m_{0}^{2} \theta\left(\varphi_{k}\right)\right)$.

$$
\begin{aligned}
\left(W_{2} \circ \varphi_{k}\right) \frac{\partial}{\partial W_{1}} & =\left(\sum_{i=1}^{k-1} v_{i} y^{i}\right) \frac{\partial}{\partial W_{1}} \\
& \equiv-\sum_{i=1}^{k-2}\left(V_{i} \circ \varphi_{k}\right) \frac{\partial}{\partial U_{i}} \quad\left(\bmod T \mathcal{R}_{e}\left(\varphi_{k}\right)+\varphi_{k}^{2} m_{0}^{2} \theta\left(\varphi_{k}\right)\right) .
\end{aligned}
$$

Thus, it follows that there exists a liftable vector field over $\varphi_{k}$ having the following form, where $\widetilde{\eta}_{2} \in m_{0}^{2} \theta_{0}(p)$.

$$
\eta_{2}+\widetilde{\eta}_{2}=\sum_{i=1}^{k-2} V_{i} \frac{\partial}{\partial U_{i}}+W_{2} \frac{\partial}{\partial W_{1}}+\text { higher terms. }
$$

Thirdly, we try to find a vector field $\eta \in m_{0} \theta_{0}(2 k-1)$ such that $W_{2} \frac{\partial}{\partial W_{2}} \neq \eta$ and $\left(W_{2} \circ \varphi_{k}\right) \frac{\partial}{\partial W_{2}} \equiv \xi \circ \varphi_{k}\left(\bmod T \mathcal{R}_{e}\left(\varphi_{k}\right)\right)$.

$$
\begin{aligned}
\left(W_{2} \circ \varphi_{k}\right) \frac{\partial}{\partial W_{2}} & =\left(\sum_{i=1}^{k-1} v_{i} y^{i}\right) \frac{\partial}{\partial W_{2}} \\
& \equiv-\sum_{i=1}^{k-1}\left(V_{i} \circ \varphi_{k}\right) \frac{\partial}{\partial V_{i}} \quad\left(\bmod T \mathcal{R}_{e}\left(\varphi_{k}\right)\right) .
\end{aligned}
$$

Thus, it follows that the following is a liftable vector field over $\varphi_{k}$ where $\widetilde{\eta}_{3}=0$.

$$
\eta_{3}+\widetilde{\eta}_{3}=\sum_{i=1}^{k-1} V_{i} \frac{\partial}{\partial V_{i}}+W_{2} \frac{\partial}{\partial W_{2}}
$$

Fourthly, since $y^{i}\left(W_{1} \circ \varphi_{k}\right) \frac{\partial}{\partial W_{1}} \equiv-\left(W_{1} \circ \varphi_{k}\right) \frac{\partial}{\partial U_{i}}\left(\bmod T \mathcal{A}_{e}\left(\varphi_{k}\right)\right)$ for any $i$ $(1 \leq i \leq k-2)$, we try to find a vector field $\eta_{i} \in m_{0} \theta_{0}(2 k-1)$ such that $-W_{1} \frac{\partial}{\partial U_{i}} \neq \eta_{i}$ and $y^{i}\left(W_{1} \circ \varphi_{k}\right) \frac{\partial}{\partial W_{1}} \equiv \eta_{i} \circ \varphi_{k}\left(\bmod T \mathcal{R}_{e}\left(\varphi_{k}\right)+\varphi_{k}^{2} m_{0}^{2} \theta\left(\varphi_{k}\right)\right)$.

$$
\begin{aligned}
& y^{i}\left(W_{1} \circ \varphi_{k}\right) \frac{\partial}{\partial W_{1}} \\
&= y^{i}\left(y^{k}+\sum_{j=1}^{k-2} u_{j} y^{j}\right) \frac{\partial}{\partial W_{1}} \\
& \equiv\left(-\frac{1}{k} \sum_{j=1}^{k-2} u_{j} y^{i+j}+\sum_{j=1}^{k-2} u_{j} y^{i+j}\right) \frac{\partial}{\partial W_{1}}-\sum_{j=1}^{k-1} j v_{j} y^{i+j} \frac{\partial}{\partial W_{2}}\left(\bmod T \mathcal{R}_{e}\left(\varphi_{k}\right)\right) . \\
& \equiv-\sum_{j=1}^{k-2-i} \frac{(k-j)}{k}\left(U_{j} \circ \varphi_{k}\right) \frac{\partial}{\partial U_{i+j}}+\sum_{j=1}^{k-1-i} j\left(V_{j} \circ \varphi_{k}\right) \frac{\partial}{\partial V_{i+j}} \\
&\left(\bmod T \mathcal{R}_{e}\left(\varphi_{k}\right)+\varphi_{k}^{2} m_{0}^{2} \theta\left(\varphi_{k}\right)\right) .
\end{aligned}
$$


Thus, it follows that for any $i(1 \leq i \leq k-2)$ there exists a liftable vector field over $\varphi_{k}$ having the following form where $\widetilde{\eta}_{3+i} \in m_{0}^{2} \theta_{0}(p)$.

$$
\begin{aligned}
& \eta_{3+i}+\widetilde{\eta}_{3+i} \\
= & W_{1} \frac{\partial}{\partial U_{i}}-\sum_{j=1}^{k-2-i} \frac{(k-j)}{k} U_{j} \frac{\partial}{\partial U_{i+j}}+\sum_{j=1}^{k-1-i} j V_{j} \frac{\partial}{\partial V_{i+j}}+\text { higher terms. }
\end{aligned}
$$

Fifthly, since $y^{i}\left(W_{2} \circ \varphi_{k}\right) \frac{\partial}{\partial W_{1}} \equiv-\left(W_{2} \circ \varphi_{k}\right) \frac{\partial}{\partial U_{i}}\left(\bmod T \mathcal{A}_{e}\left(\varphi_{k}\right)\right)$ for any $i(1 \leq$ $i \leq k-2)$, we try to find a vector field $\eta_{i} \in m_{0} \theta_{0}(2 k-1)$ such that $-W_{2} \frac{\partial}{\partial U_{i}} \neq \eta_{i}$ and $y^{i}\left(W_{2} \circ \varphi_{k}\right) \frac{\partial}{\partial W_{1}} \equiv \eta_{i} \circ \varphi_{k}\left(\bmod T \mathcal{R}_{e}\left(\varphi_{k}\right)+\varphi_{k}^{2} m_{0}^{2} \theta\left(\varphi_{k}\right)\right)$.

$$
\begin{aligned}
& y^{i}\left(W_{2} \circ \varphi_{k}\right) \frac{\partial}{\partial W_{1}} \\
& =\left(\sum_{j=1}^{k-1} v_{j} y^{i+j}\right) \frac{\partial}{\partial W_{1}} \\
& \equiv\left\{\begin{array}{rll}
-\sum_{j=1}^{k-2-i}\left(V_{j} \circ \varphi_{k}\right) \frac{\partial}{\partial U_{i+j}} & \\
& \left(\bmod T \mathcal{R}_{e}\left(\varphi_{k}\right)+\varphi_{k}^{2} m_{0}^{2} \theta\left(\varphi_{k}\right)\right) & (1 \leq i \leq k-3), \\
0 \quad & \left(\bmod T \mathcal{R}_{e}\left(\varphi_{k}\right)+\varphi_{k}^{2} m_{0}^{2} \theta\left(\varphi_{k}\right)\right) & (i=k-2)
\end{array}\right.
\end{aligned}
$$

Thus, it follows that for any $i(1 \leq i \leq k-3)$ there exists a liftable vector field over $\varphi_{k}$ having the following form where $\widetilde{\eta}_{k+1+i} \in m_{0}^{2} \theta_{0}(p)$,

$$
\eta_{k+1+i}+\widetilde{\eta}_{k+1+i}=W_{2} \frac{\partial}{\partial U_{i}}-\sum_{j=1}^{k-2-i} V_{j} \frac{\partial}{\partial U_{i+j}}+\text { higher terms }
$$

and there exists a liftable vector field over $\varphi_{k}$ having the following form, where $\widetilde{\eta}_{2 k-1} \in m_{0}^{2} \theta_{0}(p)$.

$$
\eta_{2 k-1}+\widetilde{\eta}_{2 k-1}=W_{2} \frac{\partial}{\partial U_{k-2}}+\text { higher terms. }
$$

Sixthly, since $y^{i}\left(W_{2} \circ \varphi_{k}\right) \frac{\partial}{\partial W_{2}} \equiv-\left(W_{2} \circ \varphi_{k}\right) \frac{\partial}{\partial V_{i}}\left(\bmod T \mathcal{A}_{e}\left(\varphi_{k}\right)\right)$ for any $i(1 \leq$ $i \leq k-1)$, we try to find a vector field $\eta_{i} \in m_{0} \theta_{0}(2 k-1)$ such that $-W_{2} \frac{\partial}{\partial V_{i}} \neq \eta_{i}$ and $y^{i}\left(W_{2} \circ \varphi_{k}\right) \frac{\partial}{\partial W_{2}} \equiv \eta_{i} \circ \varphi_{k}\left(\bmod T \mathcal{R}_{e}\left(\varphi_{k}\right)+\varphi_{k}^{2} m_{0}^{2} \theta\left(\varphi_{k}\right)\right)$.

$$
\begin{aligned}
& y^{i}\left(W_{2} \circ \varphi_{k}\right) \frac{\partial}{\partial W_{2}} \\
& =\left(\sum_{j=1}^{k-1} v_{j} y^{i+j}\right) \frac{\partial}{\partial W_{2}}
\end{aligned}
$$

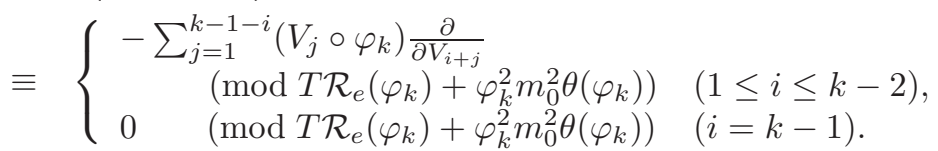

Thus, it follows that for any $i(1 \leq i \leq k-2)$ there exists a liftable vector field over $\varphi_{k}$ having the following form where $\widetilde{\eta}_{2 k-1+i} \in m_{0}^{2} \theta_{0}(p)$,

$$
\eta_{2 k-1+i}+\widetilde{\eta}_{2 k-1+i}=W_{2} \frac{\partial}{\partial V_{i}}-\sum_{j=1}^{k-1-i} V_{j} \frac{\partial}{\partial V_{i+j}}+\text { higher terms }
$$


and there exists a liftable vector field over $\varphi_{k}$ having the following form where $\widetilde{\eta}_{3 k-2} \in m_{0}^{2} \theta_{0}(p)$.

$$
\eta_{3 k-2}+\widetilde{\eta}_{3 k-2}=W_{2} \frac{\partial}{\partial V_{k-1}}+\text { higher terms. }
$$

Finally, set

$$
\begin{aligned}
\Pi=\mathbb{K} W_{1} & \frac{\partial}{\partial W_{1}}+\mathbb{K} W_{2} \frac{\partial}{\partial W_{1}}+\mathbb{K} W_{2} \frac{\partial}{\partial W_{2}} \\
& +\sum_{i=1}^{k-2} \mathbb{K} W_{1} \frac{\partial}{\partial U_{i}}+\sum_{i=1}^{k-2} \mathbb{K} W_{2} \frac{\partial}{\partial U_{i}}+\sum_{i=1}^{k-1} \mathbb{K} W_{2} \frac{\partial}{\partial V_{i}} .
\end{aligned}
$$

Then, $\Pi$ is a $(3 k-2)$-dimensional $\mathbb{K}$-vector space. Let $\pi: \theta_{0}(p) \rightarrow \Pi$ be the canonical projection. Then, we see easily that $\pi\left(\eta_{i}+\widetilde{\eta}_{i}\right)(1 \leq i \leq 3 k-2)$ constitute a basis of $\Pi$. Thus, $\eta_{i}+\widetilde{\eta}_{i}(1 \leq i \leq 3 k-2)$ constitute a set of generators for the module of vector fields liftable over $\varphi_{k}$.

6.2. $\operatorname{Lift}\left(\psi_{n}\right)$ for $\psi_{n}\left(v_{1}, \ldots, v_{n-1}, y\right)=\left(v_{1}, \ldots, v_{n-1}, y^{2}, v_{1} y, \ldots, v_{n-1} y\right)$.

We let $\left(V_{1}, \ldots, V_{n-1}, W, X_{1}, \ldots, X_{n-1}\right)$ be the standard coordinates of $\mathbb{K}^{2 n-1}$. Since ${ }_{0} \bar{\omega} \psi_{n}$ is bijective we first look for a basis of $\operatorname{ker}\left({ }_{1} \bar{\omega} \psi_{n}\right)$. We can find out easily a basis of $\operatorname{ker}\left({ }_{1} \bar{\omega} \psi_{n}\right)$ which is (for instance) the following:

$$
\begin{aligned}
& V_{i} \frac{\partial}{\partial V_{j}}+X_{i} \frac{\partial}{\partial X_{j}}+m_{0}^{2} \theta_{0}(2 n-1) \quad(1 \leq i, j \leq n-1), \\
& X_{i} \frac{\partial}{\partial V_{j}}+m_{0}^{2} \theta_{0}(2 n-1) \quad(1 \leq i, j \leq n-1), \\
& 2 X_{i} \frac{\partial}{\partial W}+m_{0}^{2} \theta_{0}(2 n-1) \quad(1 \leq i \leq n-1), \\
& 2 W \frac{\partial}{\partial W}+\sum_{j=1}^{n-1} X_{j} \frac{\partial}{\partial X_{j}}+m_{0}^{2} \theta_{0}(2 n-1) .
\end{aligned}
$$

Since any component function of $\psi_{n}$ is a monomial, we can determine easily the desired higher terms of liftable vector fields and thus we see that the following constitute a set of generators for the module of vector fields liftable over $\psi_{n}$.

$$
\begin{aligned}
& V_{i} \frac{\partial}{\partial V_{j}}+X_{i} \frac{\partial}{\partial X_{j}} \quad(1 \leq i, j \leq n-1), \\
& X_{i} \frac{\partial}{\partial V_{j}}+V_{i} W \frac{\partial}{\partial X_{j}} \quad(1 \leq i, j \leq n-1), \\
& 2 X_{i} \frac{\partial}{\partial W}+\sum_{j=1}^{n-1} V_{i} V_{j} \frac{\partial}{\partial X_{j}} \quad(1 \leq i \leq n-1), \\
& 2 W \frac{\partial}{\partial W}+\sum_{j=1}^{n-1} X_{j} \frac{\partial}{\partial X_{j}} .
\end{aligned}
$$

6.3. $\operatorname{Lift}(\phi)$ for $\phi(x, y)=\left(x, y^{2}, y^{3}+x y\right)$.

Let $(x, y),(V, W, X)$ be the standard coordinates of $\mathbb{K}^{2}$ and $\mathbb{K}^{3}$ respectively, and let $\phi:\left(\mathbb{K}^{2}, 0\right) \rightarrow\left(\mathbb{K}^{3}, 0\right)$ be the mono-germ defined by

$$
\phi(x, y)=\left(x, y^{2}, y^{3}+x y\right) .
$$


Set,

$$
h(x, y)=\left(x+y^{2}, y\right) \quad \text { and } \quad H(V, W, X)=(V-W, W, X) .
$$

Then, both $h: \mathbb{K}^{2} \rightarrow \mathbb{K}^{2}$ and $H: \mathbb{K}^{3} \rightarrow \mathbb{K}^{3}$ are analytic diffeomorphisms and preserve the origin. Moreover, we have the following:

$$
\phi(x, y)=H \circ \psi_{2} \circ h(x, y),
$$

where $\psi_{2}$ is the mono-germ defined in Example 3.3 By this equality, $f$ is $\mathcal{A}$ equivalent to $\psi_{2}$. As same as $\psi_{2}, f$ is often used as the normal form of Whitney umbrella from $\mathbb{K}^{2}$ to $\mathbb{K}^{3}$. By Subsection 6.2, we have the following:

$$
\operatorname{Lift}\left(\psi_{2}\right)=\left\langle V \frac{\partial}{\partial V}+X \frac{\partial}{\partial X}, X \frac{\partial}{\partial V}+V W \frac{\partial}{\partial X}, 2 X \frac{\partial}{\partial W}+V^{2} \frac{\partial}{\partial X}, 2 W \frac{\partial}{\partial W}+X \frac{\partial}{\partial X}\right\rangle_{C_{0}} .
$$

Thus, by using the following lemma, $\operatorname{Lift}(\phi)$ can be characterized as the $C_{0}$-module generated by the following 4 vector fields:

$$
\begin{gathered}
(V+W) \frac{\partial}{\partial V}+X \frac{\partial}{\partial X}, X \frac{\partial}{\partial V}+(V+W) W \frac{\partial}{\partial X}, \\
-2 X \frac{\partial}{\partial V}+2 X \frac{\partial}{\partial W}+(V+W)^{2} \frac{\partial}{\partial X},-2 W \frac{\partial}{\partial V}+2 W \frac{\partial}{\partial W}+X \frac{\partial}{\partial X} .
\end{gathered}
$$

Lemma 6.1. Let $S$ be a finite subset $\left\{s_{1}, \ldots, s_{r}\right\} \quad\left(s_{i} \neq s_{j}\right.$ if $\left.i \neq j\right)$ and let $f=\left\{f_{1}, \ldots, f_{r}\right\}, g=\left\{g_{1}, \ldots, g_{r}\right\}:\left(\mathbb{K}^{n}, S\right) \rightarrow\left(\mathbb{K}^{p}, 0\right)$ be two analytic multigerms. Suppose that there exist germs of analytic diffeomorphisms $h_{i}:\left(\mathbb{K}^{n}, s_{i}\right) \rightarrow\left(\mathbb{K}^{n}, s_{i}\right)$ $(1 \leq i \leq r)$ and $H:\left(\mathbb{K}^{p}, 0\right) \rightarrow\left(\mathbb{K}^{p}, 0\right)$ such that $g=H \circ f \circ h$, where $h:\left(\mathbb{K}^{n}, S\right) \rightarrow$ $\left(\mathbb{K}^{n}, S\right)$ is the map-germ whose restriction to $\left(\mathbb{K}^{n}, s_{i}\right)$ is $h_{i}$. Then, the mapping $L_{(f, g)}: \operatorname{Lift}(f) \rightarrow \operatorname{Lift}(g)$ defined by $L_{(f, g)}(\eta)=d H \circ \eta \circ H^{-1}$ is well-defined and bijective.

Proof of Lemma 6.1

Let $\eta$ be a liftable vector field over $f$. By definition, there exists $\xi \in \theta_{S}(n)$ such that $\eta \circ f=t f \circ \xi$. Since the equality $g=H \circ f \circ h$ holds, we have the following:

$$
\eta \circ\left(H^{-1} \circ g \circ h^{-1}\right)=t\left(H^{-1} \circ g \circ h^{-1}\right) \circ \xi .
$$

Hence, we have the following:

$$
\left(d H \circ \eta \circ H^{-1}\right) \circ g=t g \circ\left(d h^{-1} \circ \xi \circ h\right) .
$$

This shows that $\left(d H \circ \eta \circ H^{-1}\right)$ is a liftable vector field over $g$. Hence, the mapping $L_{(f, g)}$ is well-defined.

Since injectivity of $L_{(f, g)}$ is clear, it is sufficient to show that $L_{(f, g)}$ is surjective. Let $\widetilde{\eta}$ be a liftable vector field of $g$. The above argument shows that $d\left(H^{-1}\right) \circ \widetilde{\eta} \circ H$ is a liftable vector field of $f$. Since $L_{(f, g)}\left(d\left(H^{-1}\right) \circ \widetilde{\eta} \circ H\right)=\widetilde{\eta}$, it follows that $L_{(f, g)}$ is surjective.

6.4. Lift $(f)$ for $f(x, y)=\left\{\left(x, y^{2}\right),\left(x^{2}, y\right)\right\}$.

Let $(X, Y)$ be the standard coordinates of $\mathbb{K}^{2}$. Since ${ }_{0} \bar{\omega} f$ is bijective we first look for a basis of $\operatorname{ker}\left({ }_{1} \bar{\omega} f\right)$. We can find out easily a basis of $\operatorname{ker}\left({ }_{1} \bar{\omega} f\right)$ which is (for instance) the following:

$$
X \frac{\partial}{\partial X}+m_{0}^{2} \theta_{0}(2), Y \frac{\partial}{\partial Y}+m_{0}^{2} \theta_{0}(2)
$$


Since any component function of $f$ is a monomial, we can determine easily the desired higher terms of liftable vector fields and thus we see that the following constitute a set of generators for the module of vector fields liftable over $f$.

$$
X \frac{\partial}{\partial X}, Y \frac{\partial}{\partial Y} \text {. }
$$

6.5. Lift $(f)$ for $f(x)=\left\{\left(x^{2}, x^{3}\right),\left(x^{3}, x^{2}\right)\right\}$.

Recall that the multigerm $f$ of Example 3.6. 2 is $f_{1}(x)=\left(x^{2}, x^{3}\right), f_{2}(x)=\left(x^{3}, x^{2}\right)$. Let $(X, Y)$ be the standard coordinates of $\mathbb{K}^{2}$. Since ${ }_{1} \bar{\omega} f$ is bijective we first look for a basis of $\operatorname{ker}\left({ }_{2} \bar{\omega} f\right)$. We can find out easily a basis of $\operatorname{ker}\left({ }_{2} \bar{\omega} f\right)$ which is (for instance) the following:

$$
6 X Y \frac{\partial}{\partial X}+4 Y^{2} \frac{\partial}{\partial Y}+m_{0}^{3} \theta_{0}(2), 4 X^{2} \frac{\partial}{\partial X}+6 X Y \frac{\partial}{\partial Y}+m_{0}^{3} \theta_{0}(2) .
$$

Set $\xi_{1,1,1}=3 x^{4} \frac{\partial}{\partial x}, \xi_{1,2,1}=2 x^{3} \frac{\partial}{\partial x}$ and $\eta_{1,1}=6 X Y \frac{\partial}{\partial X}+4 Y^{2} \frac{\partial}{\partial Y}$. Then, we have the following:

$$
\begin{aligned}
& \eta_{1,1} \circ f_{1}-d f_{1} \circ \xi_{1,1,1}=-5 x^{6} \frac{\partial}{\partial Y}, \\
& \eta_{1,1} \circ f_{2}-d f_{2} \circ \xi_{1,2,1}=0 .
\end{aligned}
$$

Set $\eta_{1,2}=5 X^{3} \frac{\partial}{\partial Y}$. Then we have the following:

$$
\begin{gathered}
\left(\eta_{1,1}+\eta_{1,2}\right) \circ f_{1}-d f_{1} \circ \xi_{1,1,1}=0, \\
\left(\eta_{1,1}+\eta_{1,2}\right) \circ f_{2}-d f_{2} \circ \xi_{1,2,1}=5 x^{9} \frac{\partial}{\partial Y} .
\end{gathered}
$$

Set $\eta_{1,3}=-5 X Y^{3} \frac{\partial}{\partial Y}$ and $\xi_{1,1,2}=-\frac{5}{3} x^{9} \frac{\partial}{\partial x}$. Then we have the following:

$$
\begin{aligned}
& \left(\eta_{1,1}+\eta_{1,2}+\eta_{1,3}\right) \circ f_{1}-d f_{1} \circ\left(\xi_{1,1,1}+\xi_{1,1,2}\right)=\frac{10}{3} x^{10} \frac{\partial}{\partial X}, \\
& \left(\eta_{1,1}+\eta_{1,2}+\eta_{1,3}\right) \circ f_{2}-d f_{2} \circ \xi_{1,2,1}=0 .
\end{aligned}
$$

Set $\eta_{1,4}=-\frac{10}{3} X^{2} Y^{2} \frac{\partial}{\partial X}$ and $\xi_{1,2,2}=-\frac{10}{9} x^{8} \frac{\partial}{\partial x}$. Then we have the following:

$$
\begin{gathered}
\left(\eta_{1,1}+\eta_{1,2}+\eta_{1,3}+\eta_{1,4}\right) \circ f_{1}-d f_{1} \circ\left(\xi_{1,1,1}+\xi_{1,1,2}\right)=0, \\
\left(\eta_{1,1}+\eta_{1,2}+\eta_{1,3}+\eta_{1,4}\right) \circ f_{2}-d f_{2} \circ\left(\xi_{1,2,1}+\xi_{1,2,2}\right)=\frac{20}{9} x^{9} \frac{\partial}{\partial Y} .
\end{gathered}
$$

Note that the right hand side of (5.3) (resp., the right hand side of (5.4)) is the right hand side of (5.1) (resp., the right hand side of (5.2)) multiplied by $\left(\frac{2}{3}\right)^{2}$. Thus, the following vector field $\eta_{1}$ must be liftable over $f$.

$$
\begin{aligned}
\eta_{1} & =\eta_{1,1}+\eta_{1,2}+\left(1+\left(\frac{2}{3}\right)^{2}+\left(\frac{2}{3}\right)^{4}+\cdots\right)\left(\eta_{1,3}+\eta_{1,4}\right) \\
& =\left(6 X Y-6 X^{2} Y^{2}\right) \frac{\partial}{\partial X}+\left(4 Y^{2}+5 X^{3}-9 X Y^{3}\right) \frac{\partial}{\partial Y} .
\end{aligned}
$$

Next, Set $\xi_{2,1,1}=2 x^{3} \frac{\partial}{\partial x}, \xi_{2,2,1}=3 x^{4} \frac{\partial}{\partial x}$ and $\eta_{2,1}=4 X^{2} \frac{\partial}{\partial X}+6 X Y \frac{\partial}{\partial Y}$. Then, we have the following:

$$
\begin{aligned}
& \eta_{2,1} \circ f_{1}-d f_{1} \circ \xi_{2,1,1}=0, \\
& \eta_{2,1} \circ f_{2}-d f_{2} \circ \xi_{2,2,1}=-5 x^{6} \frac{\partial}{\partial X} .
\end{aligned}
$$


Set $\eta_{2,2}=5 Y^{3} \frac{\partial}{\partial X}$. Then we have the following:

$$
\begin{gathered}
\left(\eta_{2,1}+\eta_{2,2}\right) \circ f_{1}-d f_{1} \circ \xi_{2,1,1}=5 x^{9} \frac{\partial}{\partial X}, \\
\left(\eta_{2,1}+\eta_{2,2}\right) \circ f_{2}-d f_{2} \circ \xi_{2,2,1}=0 .
\end{gathered}
$$

Set $\eta_{2,3}=-5 X^{3} Y \frac{\partial}{\partial X}$ and $\xi_{2,2,2}=-\frac{5}{3} x^{9} \frac{\partial}{\partial x}$. Then we have the following:

$$
\begin{aligned}
& \left(\eta_{2,1}+\eta_{2,2}+\eta_{2,3}\right) \circ f_{1}-d f_{1} \circ\left(\xi_{2,1,1}\right)=0, \\
& \left(\eta_{2,1}+\eta_{2,2}+\eta_{2,3}\right) \circ f_{2}-d f_{2} \circ\left(\xi_{2,2,1}+\xi_{2,2,2}\right)=\frac{10}{3} x^{10} \frac{\partial}{\partial Y} .
\end{aligned}
$$

Set $\eta_{2,4}=-\frac{10}{3} X^{2} Y^{2} \frac{\partial}{\partial Y}$ and $\xi_{2,1,2}=-\frac{10}{9} x^{8} \frac{\partial}{\partial x}$. Then we have the following:

$$
\begin{gathered}
\left(\eta_{2,1}+\eta_{2,2}+\eta_{2,3}+\eta_{2,4}\right) \circ f_{1}-d f_{1} \circ\left(\xi_{2,1,1}+\xi_{2,1,2}\right)=\frac{20}{9} x^{9} \frac{\partial}{\partial X}, \\
\left(\eta_{2,1}+\eta_{2,2}+\eta_{2,3}+\eta_{2,4}\right) \circ f_{2}-d f_{2} \circ\left(\xi_{2,2,1}+\xi_{2,2,2}\right)=0 .
\end{gathered}
$$

Note that the right hand side of (5.7) (resp., the right hand side of (5.8)) is the right hand side of (5.5) (resp., the right hand side of (5.6)) multiplied by $\left(\frac{2}{3}\right)^{2}$. Thus, the following vector field $\eta_{2}$ must be liftable over $f$.

$$
\begin{aligned}
\eta_{2} & =\eta_{2,1}+\eta_{2,2}+\left(1+\left(\frac{2}{3}\right)^{2}+\left(\frac{2}{3}\right)^{4}+\cdots\right)\left(\eta_{2,3}+\eta_{2,4}\right) \\
& =\left(4 X^{2}+5 Y^{3}-9 X^{3} Y\right) \frac{\partial}{\partial X}+\left(6 X Y-6 X^{2} Y^{2}\right) \frac{\partial}{\partial Y} .
\end{aligned}
$$

Therefore, the following constitute a set of generators for the module of vector fields liftable over $f$.

$$
\begin{aligned}
& \eta_{1}=\left(6 X Y-6 X^{2} Y^{2}\right) \frac{\partial}{\partial X}+\left(4 Y^{2}+5 X^{3}-9 X Y^{3}\right) \frac{\partial}{\partial Y} \\
& \eta_{2}=\left(4 X^{2}+5 Y^{3}-9 X^{3} Y\right) \frac{\partial}{\partial X}+\left(6 X Y-6 X^{2} Y^{2}\right) \frac{\partial}{\partial Y} .
\end{aligned}
$$

6.6. Lift $(f)$ for $f(y)=\left(y^{2}, 0\right)$.

Let $f:(\mathbb{K}, 0) \rightarrow\left(\mathbb{K}^{2}, 0\right)$ be the mono-germ defined by $f(y)=\left(y^{2}, 0\right)$. As an application of Theorem 2, we obtain all liftable vector fields over $f$.

Let $(Y, U)$ be the standard coordinates of the target space of $f$. It is easy to see the following:

$$
\theta_{S}(f)=T \mathcal{K}_{e}(f)+\mathbb{K}^{2}+y \frac{\partial}{\partial U}
$$

Since $\operatorname{dim}_{\mathbb{K}} \theta_{S}(f) /\left(T \mathcal{K}_{e}(f)+\mathbb{K}^{2}\right)=1$, by Mather's constructing method of stable mono-germs (15), the mono-germ $F(x, y)=\left(x, y^{2}, x y\right)$ is a one-parameter stable unfolding of $f$. Notice that $F$ is exactly the same as the mono-germ $\psi_{2}$ defined in Subsection 6.2. Let $(X, Y, U)$ be the standard coordinates of the target space of $F$. Let $g:\left(\mathbb{K} \times \mathbb{K}^{2},(0,0)\right) \rightarrow\left(\mathbb{K} \times \mathbb{K}^{2},(0,0)\right)$ be defined by $g(x, y, u)=\left(x^{2}, y, u\right)$. Then, $\operatorname{Lift}(g)=\left\langle X \frac{\partial}{\partial X}, \frac{\partial}{\partial Y}, \frac{\partial}{\partial U}\right\rangle_{C_{0}}$. Set $\widetilde{\eta}_{1}=X \frac{\partial}{\partial X}+U \frac{\partial}{\partial U}, \widetilde{\eta}_{2}=U \frac{\partial}{\partial X}+X Y \frac{\partial}{\partial U}$, $\widetilde{\eta}_{3}=2 U \frac{\partial}{\partial Y}+X^{2} \frac{\partial}{\partial U}$ and $\widetilde{\eta}_{4}=2 Y \frac{\partial}{\partial Y}+U \frac{\partial}{\partial U}$. By Subsection 6.2 we have the 
following:

$$
\begin{aligned}
\operatorname{Lift}(F)= & \left\langle\widetilde{\eta}_{1}, \widetilde{\eta}_{2}, \widetilde{\eta}_{3}, \widetilde{\eta}_{4}\right\rangle_{C_{0}} \\
= & \left\{\widetilde{\alpha}_{1}\left(X \frac{\partial}{\partial X}+U \frac{\partial}{\partial U}\right)+\widetilde{\alpha}_{2}\left(U \frac{\partial}{\partial X}+X Y \frac{\partial}{\partial U}\right)\right. \\
& \left.\quad+\widetilde{\alpha}_{3}\left(2 U \frac{\partial}{\partial Y}+X^{2} \frac{\partial}{\partial U}\right)+\widetilde{\alpha_{4}}\left(2 Y \frac{\partial}{\partial Y}+U \frac{\partial}{\partial U}\right)\right\},
\end{aligned}
$$

where $\widetilde{\alpha}_{i}(1 \leq i \leq 4)$ are analytic function-germs of three variables $X, Y, U$. Thus, we have the following:

$$
\begin{aligned}
& \quad \operatorname{Lift}(F) \cap \operatorname{Lift}(g) \\
& =\left\{\widetilde{\alpha}_{1}\left(X \frac{\partial}{\partial X}+U \frac{\partial}{\partial U}\right)+\widetilde{\alpha}_{2}\left(U \frac{\partial}{\partial X}+X Y \frac{\partial}{\partial U}\right)+\widetilde{\alpha}_{3}\left(2 U \frac{\partial}{\partial Y}+X^{2} \frac{\partial}{\partial U}\right)\right. \\
& \left.+\widetilde{\alpha}_{4}\left(2 Y \frac{\partial}{\partial Y}+U \frac{\partial}{\partial U}\right) \mid \widetilde{\alpha}_{1} X+\widetilde{\alpha}_{2} U \text { can be divided by } X\right\} \\
& =\left\{\widetilde{\alpha}_{1}\left(X \frac{\partial}{\partial X}+U \frac{\partial}{\partial U}\right)+\widetilde{\alpha}_{2}\left(U \frac{\partial}{\partial X}+X Y \frac{\partial}{\partial U}\right)+\widetilde{\alpha}_{3}\left(2 U \frac{\partial}{\partial Y}+X^{2} \frac{\partial}{\partial U}\right)\right. \\
& \left.+\widetilde{\alpha}_{4}\left(2 Y \frac{\partial}{\partial Y}+U \frac{\partial}{\partial U}\right) \mid \widetilde{\alpha}_{2} \text { can be divided by } X\right\} .
\end{aligned}
$$

Define $\alpha_{i}:\left(\mathbb{K}^{2}, 0\right) \rightarrow \mathbb{K}(1 \leq i \leq 4)$ by $\alpha_{i}(Y, U)=\widetilde{\alpha}_{i}(0, Y, U)$. Then, by Theorem 2. we have the following:

$$
\begin{aligned}
& \operatorname{Lift}(f) \\
= & \left\{\alpha_{1} U \frac{\partial}{\partial U}+2 \alpha_{3} U \frac{\partial}{\partial Y}+\alpha_{4}\left(2 Y \frac{\partial}{\partial Y}+U \frac{\partial}{\partial U}\right)\right\} \\
= & \left\langle U \frac{\partial}{\partial U}, U \frac{\partial}{\partial Y}, Y \frac{\partial}{\partial Y}\right\rangle_{C_{0}} .
\end{aligned}
$$

Since three vector fields $U \frac{\partial}{\partial U}, U \frac{\partial}{\partial Y}, Y \frac{\partial}{\partial Y}$ are linearly independent, the minimal number of generators for $\operatorname{Lift}(f)$ is 3 , which is strictly greater than the dimension of the target space of $f$.

6.7. Lift $\left(f_{k}\right)$ for $f_{k}(y)=\left(y^{2}, y^{2 k+1}\right)(k \geq 1)$.

Let $f_{k}:(\mathbb{K}, 0) \rightarrow\left(\mathbb{K}^{2}, 0\right)(k \geq 1)$ be the mono-germ defined by $f_{k}(y)=\left(y^{2}, y^{2 k+1}\right)$. As an application of Theorem 2 we obtain all liftable vector fields over $f_{k}$.

Let $(Y, U)$ be the standard coordinates of the target space of $f_{k}$. It is easy to see the following:

$$
\theta_{S}\left(f_{k}\right)=T \mathcal{K}_{e}\left(f_{k}\right)+\mathbb{K}^{2}+y \frac{\partial}{\partial U}
$$

Since $\operatorname{dim}_{\mathbb{K}} \theta_{S}\left(f_{k}\right) /\left(T \mathcal{K}_{e}\left(f_{k}\right)+\mathbb{K}^{2}\right)=1$, by Mather's constructing method of stable mono-germs $([15])$, the mono-germ $F_{k}(x, y)=\left(x, y^{2}, y^{2 k+1}+x y\right)$ is a one-parameter stable unfolding of $f_{k}$. Set $F(x, y)=\left(x, y^{2}, x y\right)$. Let $(X, Y, U)$ be the standard coordinates of the target space of $F_{k}$. Set $h_{k}(x, y)=\left(x+y^{2 k}, y\right)$ and $H_{k}(X, Y, U)=$ $\left(X-Y^{k}, Y, U\right)$. Then, both $h_{k}$ and $H_{k}$ are analytic diffeomorphisms preserving the origin, and we have that $F_{k}=H_{k} \circ F \circ h_{k}$. Set $\widetilde{\eta}_{1}=X \frac{\partial}{\partial X}+U \frac{\partial}{\partial U}, \widetilde{\eta}_{2}=$ $U \frac{\partial}{\partial X}+X Y \frac{\partial}{\partial U}, \widetilde{\eta}_{3}=2 U \frac{\partial}{\partial Y}+X^{2} \frac{\partial}{\partial U}$ and $\widetilde{\eta}_{4}=2 Y \frac{\partial}{\partial Y}+U \frac{\partial}{\partial U}$. By Subsection 6.2. we have the following:

$$
\operatorname{Lift}(F)=\left\langle\widetilde{\eta}_{1}, \widetilde{\eta}_{2}, \widetilde{\eta}_{3}, \widetilde{\eta}_{4}\right\rangle_{C_{0}}
$$


Set $\eta_{1}=d H_{k} \circ \widetilde{\eta}_{1} \circ H_{k}^{-1}, \eta_{2}=d H_{k} \circ \widetilde{\eta}_{2} \circ H_{k}^{-1}, \eta_{3}=d H_{k} \circ \widetilde{\eta}_{3} \circ H_{k}^{-1}$ and $\eta_{4}=$ $d H_{k} \circ \widetilde{\eta}_{4} \circ H_{k}^{-1}$. By Lemma 6.1 we have the following:

$$
\operatorname{Lift}\left(F_{k}\right)=\left\langle\eta_{1}, \eta_{2}, \eta_{3}, \eta_{4}\right\rangle_{C_{0}} .
$$

By calculations, we have the following:

$$
\left\{\begin{array}{l}
\eta_{1}(X, Y, U)=\left(X+Y^{k}\right) \frac{\partial}{\partial X}+U \frac{\partial}{\partial U} \\
\eta_{2}(X, Y, U)=U \frac{\partial}{\partial X}+\left(X+Y^{k}\right) Y \frac{\partial}{\partial U} \\
\eta_{3}(X, Y, U)=-2 k Y^{k-1} U \frac{\partial}{\partial X}+2 U \frac{\partial}{\partial Y}+\left(X+Y^{k}\right)^{2} \frac{\partial}{\partial U} \\
\eta_{4}(X, Y, U)=-2 k Y^{k} \frac{\partial}{\partial X}+2 Y \frac{\partial}{\partial Y}+U \frac{\partial}{\partial U} .
\end{array}\right.
$$

Let $g:\left(\mathbb{K} \times \mathbb{K}^{2},(0,0)\right) \rightarrow\left(\mathbb{K} \times \mathbb{K}^{2},(0,0)\right)$ be defined by $g(x, y, u)=\left(x^{2}, y, u\right)$.

Then, $\operatorname{Lift}(g)=\left\langle X \frac{\partial}{\partial X}, \frac{\partial}{\partial Y}, \frac{\partial}{\partial U}\right\rangle_{C_{0}}$. Thus, we have the following:

$$
\begin{aligned}
& \operatorname{Lift}\left(F_{k}\right) \cap \operatorname{Lift}(g) \\
= & \left\{\sum_{i=1}^{4} \widetilde{\alpha}_{i} \eta_{i} \mid \widetilde{\alpha}_{1}\left(X+Y^{k}\right)+\widetilde{\alpha}_{2} U-2 k \widetilde{\alpha}_{3} Y^{k-1} U-2 k \widetilde{\alpha}_{4} Y^{k} \text { can be divided by } X\right\},
\end{aligned}
$$

where $\widetilde{\alpha}_{i}(1 \leq i \leq 4)$ are analytic function-germs of three variables $X, Y, U$. Define $\alpha_{i}:\left(\mathbb{K}^{2}, 0\right) \rightarrow \mathbb{K}(1 \leq i \leq 4)$ by $\alpha_{i}(Y, U)=\widetilde{\alpha}_{i}(0, Y, U)$. Then, by Theorem 2 $\operatorname{Lift}\left(f_{k}\right)$ can be characterized as follows:

$$
\begin{aligned}
& \operatorname{Lift}\left(f_{k}\right) \\
& =\left\{2\left(U \alpha_{3}+Y \alpha_{4}\right) \frac{\partial}{\partial Y}\right. \\
& \left.+\left(U\left(\alpha_{1}+\alpha_{4}\right)+Y^{2 k} \alpha_{3}+Y^{k+1} \alpha_{2}\right) \frac{\partial}{\partial U} \mid Y^{k} \alpha_{1}+U \alpha_{2}-2 k Y^{k-1} U \alpha_{3}-2 k Y^{k} \alpha_{4}=0\right\} \\
& =\left\{2\left(U \alpha_{3}+Y \alpha_{4}\right) \frac{\partial}{\partial Y}\right. \\
& \left.+\left(U\left(\alpha_{1}+\alpha_{4}\right)+Y^{2 k} \alpha_{3}+Y^{k+1} \alpha_{2}\right) \frac{\partial}{\partial U} \mid Y^{k}\left(\alpha_{1}-2 k \alpha_{4}\right)+U\left(\alpha_{2}-2 k Y^{k-1} \alpha_{3}\right)=0\right\} \\
& =\left\{2\left(U \alpha_{3}+Y \alpha_{4}\right) \frac{\partial}{\partial Y}\right. \\
& \left.+\left(U\left(\alpha_{1}+\alpha_{4}\right)+Y^{2 k} \alpha_{3}+Y^{k+1} \alpha_{2}\right) \frac{\partial}{\partial U} \mid \alpha_{1}-2 k \alpha_{4}=U \beta, \alpha_{2}-2 k Y^{k-1} \alpha_{3}=-Y^{k} \beta\right\} \\
& \left.=\left\{2\left(U \alpha_{3}+Y \alpha_{4}\right) \frac{\partial}{\partial Y}+\left(U(1+2 k) \alpha_{4}+U^{2} \beta\right)+Y^{2 k}(1+2 k) \alpha_{3}-Y^{2 k+1} \beta\right) \frac{\partial}{\partial U}\right\} \\
& =\left\{\alpha_{3}\left(2 U \frac{\partial}{\partial Y}+(1+2 k) Y^{2 k} \frac{\partial}{\partial U}\right)\right. \\
& \left.+\alpha_{4}\left(2 Y \frac{\partial}{\partial Y}+(1+2 k) U \frac{\partial}{\partial U}\right)+\beta\left(U^{2}-Y^{2 k+1}\right) \frac{\partial}{\partial U}\right\} \\
& =\left\{\left(\alpha_{3}-\frac{1}{1+2 k} Y \beta\right)\left(2 U \frac{\partial}{\partial Y}+(1+2 k) Y^{2 k} \frac{\partial}{\partial U}\right)\right. \\
& \left.+\left(\alpha_{4}+\frac{1}{1+2 k} U \beta\right)\left(2 Y \frac{\partial}{\partial Y}+(1+2 k) U \frac{\partial}{\partial U}\right)\right\} \\
& =\left\langle 2 U \frac{\partial}{\partial Y}+(1+2 k) Y^{2 k} \frac{\partial}{\partial U}, 2 Y \frac{\partial}{\partial Y}+(1+2 k) U \frac{\partial}{\partial U}\right\rangle_{C_{0}},
\end{aligned}
$$


where $\beta$ is an analytic function-germ of two variables $Y, U$.

6.8. $\operatorname{Lift}\left(S_{k}^{ \pm}\right)$for $S_{k}^{ \pm}(x, y)=\left(x, y^{2}, y^{3} \pm x^{k+1} y\right)(k \geq 0)$.

Let $S_{k}^{ \pm}:\left(\mathbb{K}^{2}, 0\right) \rightarrow\left(\mathbb{K}^{3}, 0\right)$ be the mono-germ defined by $S_{k}^{ \pm}(x, y)=\left(x, y^{2}, y^{3} \pm\right.$ $\left.x^{k+1} y\right)(k \geq 0)$. The mono-germ $S_{k}^{ \pm}$can be found in the classification list of $\mathcal{A}$ simple mono-germ from the plane to 3 -space due to Mond ([19]). Here, a multigerm $f:\left(\mathbb{K}^{n}, S\right) \rightarrow\left(\mathbb{K}^{p}, 0\right)$ is said to be $\mathcal{A}$-simple if there exists a finite number of $\mathcal{A}$ equivalence classes such that for any positive integer $d$ and any analytic mapping $F: U \rightarrow V$ where $U \subset \mathbb{K}^{n} \times \mathbb{K}^{d}$ is a neighbourhood of $S \times 0, V \subset \mathbb{K}^{p} \times \mathbb{K}^{d}$ is a neighbourhood of $(0,0), F(x, \lambda)=\left(f_{\lambda}(x), \lambda\right)$ and the germ of $f_{0}$ at $S$ is $f$, there exists a sufficiently small neighbourhood $W_{i} \subset U$ of $\left(s_{i}, 0\right)(1 \leq i \leq|S|)$ such that for every $\left\{\left(x_{1}, \lambda\right), \cdots,\left(x_{r}, \lambda\right)\right\}(r \leq|S|)$ with $\left(x_{i}, \lambda\right) \in W_{i}$ and $F\left(x_{1}, \lambda\right)=\cdots=$ $F\left(x_{r}, \lambda\right)$ the multigerm $f_{\lambda}:\left(\mathbb{K}^{n},\left\{x_{1}, \cdots, x_{r}\right\}\right) \rightarrow\left(\mathbb{K}^{p}, f_{\lambda}\left(x_{i}\right)\right)$ lies in one of these finite $\mathcal{A}$-equivalence classes. As an application of Theorem 2 we obtain all liftable vector fields over $S_{k}^{ \pm}$.

Let $(X, Y, U)$ be the standard coordinates of the target space of $S_{k}^{ \pm}$. It is easy to see the following:

$$
\theta_{S}\left(S_{k}^{ \pm}\right)=T \mathcal{K}_{e}\left(S_{k}^{ \pm}\right)+\mathbb{K}^{3}+y \frac{\partial}{\partial U}
$$

Since $\left.\operatorname{dim}_{\mathbb{K}}\left(\theta_{S}\left(S_{k}^{ \pm}\right) / T \mathcal{K}_{e}\left(S_{k}^{ \pm}\right)+\mathbb{K}^{3}\right)\right) \leq 1$, by Mather's constructing method of stable mono-germs ([15), the mono-germ $F_{k}^{ \pm}(x, y, u)=\left(x, y^{2}, y^{3} \pm x^{k+1} y+u y, u\right)$ is a one-parameter stable unfolding of $S_{k}^{ \pm}$. Set $F(x, y, u)=\left(x, y^{2}, y^{3}+u y, u\right)$. Let $(X, Y, U, V)$ be the standard coordinates of the target space of $F_{k}$. Set $h_{k}^{ \pm}(x, y, u)=$ $\left(x, y, u \pm x^{k+1}\right)$ and $H_{k}^{ \pm}(X, Y, U, V)=\left(X, Y, U, V \mp X^{k+1}\right)$. Then, both $h_{k}^{ \pm}$and $H_{k}^{ \pm}$are analytic diffeomorphisms preserving the origin, and we have that $F_{k}^{ \pm}=$ $H_{k}^{ \pm} \circ F \circ h_{k}^{ \pm}$. Set $\widetilde{\eta}_{1}=U \frac{\partial}{\partial U}+(Y+V) \frac{\partial}{\partial V}, \widetilde{\eta}_{2}=(Y+V) Y \frac{\partial}{\partial U}+U \frac{\partial}{\partial V}, \widetilde{\eta}_{3}=$ $2 U \frac{\partial}{\partial Y}+(Y+V)^{2} \frac{\partial}{\partial U}-2 U \frac{\partial}{\partial V}, \widetilde{\eta}_{4}=2 Y \frac{\partial}{\partial Y}+U \frac{\partial}{\partial U}-2 Y \frac{\partial}{\partial V}$ and $\widetilde{\eta}_{5}=\frac{\partial}{\partial X}$. By calculations, we have the following:

$$
\operatorname{Lift}(F)=\left\langle\widetilde{\eta}_{1}, \widetilde{\eta}_{2}, \widetilde{\eta}_{3}, \widetilde{\eta}_{4}, \widetilde{\eta}_{5}\right\rangle_{C_{0}} .
$$

Set $\eta_{1}=d\left(H_{k}^{ \pm}\right) \circ \widetilde{\eta}_{1} \circ\left(H_{k}^{ \pm}\right)^{-1}, \eta_{2}=d\left(H_{k}^{ \pm}\right) \circ \widetilde{\eta}_{2} \circ\left(H_{k}^{ \pm}\right)^{-1}, \eta_{3}=d\left(H_{k}^{ \pm}\right) \circ \widetilde{\eta}_{3} \circ\left(H_{k}^{ \pm}\right)^{-1}$, $\eta_{4}=d\left(H_{k}^{ \pm}\right) \circ \widetilde{\eta}_{4} \circ\left(H_{k}^{ \pm}\right)^{-1}$ and $\eta_{5}=d\left(H_{k}^{ \pm}\right) \circ \widetilde{\eta}_{5} \circ\left(H_{k}^{ \pm}\right)^{-1}$. By Lemma 6.1, we have the following:

$$
\operatorname{Lift}\left(F_{k}\right)=\left\langle\eta_{1}, \eta_{2}, \eta_{3}, \eta_{4}, \eta_{5}\right\rangle_{C_{0}} .
$$

By calculations, we have the following:

$$
\left\{\begin{array}{l}
\eta_{1}(X, Y, U, V)=U \frac{\partial}{\partial U}+\left(Y+V \pm X^{k+1}\right) \frac{\partial}{\partial V} \\
\eta_{2}(X, Y, U, V)=Y\left(Y+V \pm X^{k+1}\right) \frac{\partial}{\partial Y}+U \frac{\partial}{\partial V} \\
\eta_{3}(X, Y, U, V)=2 U \frac{\partial}{\partial Y}+\left(Y+V \pm X^{k+1}\right)^{2} \frac{\partial}{\partial U}-2 U \frac{\partial}{\partial V} \\
\eta_{4}(X, Y, U, V)=2 Y \frac{\partial}{\partial Y}+U \frac{\partial}{\partial U}-2 Y \frac{\partial}{\partial V} \\
\eta_{5}(X, Y, U, V)=\frac{\partial}{\partial X} \mp(k+1) X^{k} \frac{\partial}{\partial V} .
\end{array}\right.
$$

Let $g:\left(\mathbb{K}^{3} \times \mathbb{K},(0,0)\right) \rightarrow\left(\mathbb{K}^{3} \times \mathbb{K},(0,0)\right)$ be defined by $g(x, y, u, v)=\left(x, y, u, v^{2}\right)$. Then, $\operatorname{Lift}(g)=\left\langle\frac{\partial}{\partial X}, \frac{\partial}{\partial Y}, \frac{\partial}{\partial U}, V \frac{\partial}{\partial V}\right\rangle_{C_{0}}$. Thus, we have the following:

$$
\operatorname{Lift}\left(F_{k}\right) \cap \operatorname{Lift}(g)=\left\{\sum_{i=1}^{5} \widetilde{\alpha}_{i} \eta_{i} \mid \Phi(X, Y, U, V) \text { can be divided by } V\right\} \text {, }
$$


where $\widetilde{\alpha}_{i}(1 \leq i \leq 5)$ are analytic function-germs of four variables $X, Y, U, V$ and $\Phi(X, Y, U, V)$ is given as follows:

$$
\begin{aligned}
\Phi(X, Y, U, V)=\widetilde{\alpha}_{1}( & X, Y, U, V)\left(Y+V \pm X^{k+1}\right) \\
& +\widetilde{\alpha}_{2}(X, Y, U, V) U-2 \widetilde{\alpha}_{3}(X, Y, U, V) U \\
& -2 \widetilde{\alpha}_{4}(X, Y, U, V) Y \mp(k+1) \widetilde{\alpha}_{5}(X, Y, U, V) X^{k} .
\end{aligned}
$$

Define $\alpha_{i}:\left(\mathbb{K}^{3}, 0\right) \rightarrow \mathbb{K}(1 \leq i \leq 5)$ by $\alpha_{i}(X, Y, U)=\widetilde{\alpha}_{i}(X, Y, U, 0)$. Then, by Theorem 2 any element of $\operatorname{Lift}\left(S_{k}^{ \pm}\right)$has the following form:

$$
\begin{aligned}
\alpha_{1} U \frac{\partial}{\partial U}+\alpha_{2} Y\left(Y \pm X^{k+1}\right) \frac{\partial}{\partial Y}+ & \alpha_{3}\left(2 U \frac{\partial}{\partial Y}+\left(Y \pm X^{k+1}\right)^{2} \frac{\partial}{\partial U}\right) \\
& +\alpha_{4}\left(2 Y \frac{\partial}{\partial Y}+U \frac{\partial}{\partial U}\right)+\alpha_{5} \frac{\partial}{\partial X} .
\end{aligned}
$$

And, by Theorem 2 again, the unique restriction on $\alpha_{i}(1 \leq i \leq 5)$ is as follows.

\section{Condition 6.1.}

$$
\Phi(X, Y, U, 0)=\alpha_{1}\left(Y \pm X^{k+1}\right)+\alpha_{2} U-2 \alpha_{3} U-2 \alpha_{4} Y \mp(k+1) \alpha_{5} X^{k}=0 .
$$

In the case $k=0$, by Condition 6.1] it follows that $\alpha_{5}$ can be expressed by using $\alpha_{i}(1 \leq i \leq 4)$. Thus, it is easy to obtain four vector fields which constitute a generators of $\operatorname{Lift}\left(S_{0}^{ \pm}\right)$. In the case $k \geq 1$, by Condition 6.1, we have the following expressions:

$$
\begin{aligned}
\alpha_{1}-2 \alpha_{4} & =\beta_{1} X^{k}+\gamma U \\
\alpha_{2}-2 \alpha_{3} & =\beta_{2} X^{k}-\gamma Y,
\end{aligned}
$$

where $\beta_{1}, \beta_{2}, \gamma$ are analytic function-germs $\left(\mathbb{K}^{3}, 0\right) \rightarrow \mathbb{K}$. Therefore, we have the following:

$$
\alpha_{5}= \pm \frac{1}{k+1}\left(\beta_{1} Y+\beta_{2} U \pm\left(2 \alpha_{4}+\beta_{1} X^{k}+\gamma U\right) X\right)
$$

Hence, $\operatorname{Lift}\left(S_{k}^{ \pm}\right)$in the case $k \geq 1$ can be characterized as follows:

$$
\begin{gathered}
\operatorname{Lift}\left(S_{k}^{ \pm}\right) \\
=\left\{\left(2 \alpha_{4}+\beta_{1} X^{k}+\gamma U\right) U \frac{\partial}{\partial U}+\left(2 \alpha_{3}+\beta_{2} X^{k}-\gamma Y\right)\left(Y \pm X^{k+1}\right) Y \frac{\partial}{\partial U}\right. \\
+\alpha_{3}\left(2 U \frac{\partial}{\partial Y}+\left(Y \pm X^{k+1}\right)^{2} \frac{\partial}{\partial U}\right)+\alpha_{4}\left(2 Y \frac{\partial}{\partial Y}+U \frac{\partial}{\partial U}\right) \\
\left. \pm \frac{1}{k+1}\left(\beta_{1} Y+\beta_{2} U \pm\left(2 \alpha_{4}+\beta_{1} X^{k}+\gamma U\right) X\right) \frac{\partial}{\partial X}\right\} \\
=\left\langle 2 U \frac{\partial}{\partial Y}+\left(3 Y^{2} \pm 4 X^{k+1} Y+X^{2 k+2}\right) \frac{\partial}{\partial U}, \frac{2 X}{k+1} \frac{\partial}{\partial X}+2 Y \frac{\partial}{\partial Y}+3 U \frac{\partial}{\partial U},\right. \\
\pm \frac{1}{k+1}\left(Y \pm X^{k+1}\right) \frac{\partial}{\partial X}+X^{k} U \frac{\partial}{\partial U}, \pm \frac{U}{k+1} \frac{\partial}{\partial X}+X^{k} Y\left(Y \pm X^{k+1}\right) \frac{\partial}{\partial U} \\
\left.+\frac{X U}{k+1} \frac{\partial}{\partial X}+\left(U^{2}-Y^{2}\left(Y \pm X^{k+1}\right)\right) \frac{\partial}{\partial U}\right\rangle_{C_{0}} .
\end{gathered}
$$


Set $\mathbf{v}_{1}=2 U \frac{\partial}{\partial Y}+\left(3 Y^{2} \pm 4 X^{k+1} Y+X^{2 k+2}\right) \frac{\partial}{\partial U}, \mathbf{v}_{2}=\frac{2 X}{k+1} \frac{\partial}{\partial X}+2 Y \frac{\partial}{\partial Y}+3 U \frac{\partial}{\partial U}$, $\mathbf{v}_{3}= \pm \frac{1}{k+1}\left(Y \pm X^{k+1}\right) \frac{\partial}{\partial X}+X^{k} U \frac{\partial}{\partial U}, \mathbf{v}_{4}= \pm \frac{U}{k+1} \frac{\partial}{\partial X}+X^{k} Y\left(Y \pm X^{k+1}\right) \frac{\partial}{\partial U}$ and $\mathbf{v}_{5}=\frac{X U}{k+1} \frac{\partial}{\partial X}+\left(U^{2}-Y^{2}\left(Y \pm X^{k+1}\right)\right) \frac{\partial}{\partial U}$. Then, we have the following relation:

$$
-Y \mathbf{v}_{1}+U \mathbf{v}_{2} \pm X \mathbf{v}_{4}=\mathbf{v}_{5} .
$$

And, it is easily seen that none of $\mathbf{v}_{1}, \mathbf{v}_{2}, \mathbf{v}_{3}, \mathbf{v}_{4}$ can be generated by others.

It is also easily seen that the minimal number of generators for $\operatorname{Lift}\left(S_{k}^{ \pm}\right)$is less than or equal to the minimal number of generators for $\operatorname{Lift}(F)$. Thus, the minimal number of generators for $\operatorname{Lift}\left(S_{k}^{ \pm}\right)$is less than or equal to 5 . It is interesting to observe that the minimal number of generators for $\operatorname{Lift}\left(S_{k}^{ \pm}\right)$is always 4 . It is also interesting to observe that the germs $B_{k}^{ \pm}, C_{k}^{ \pm}$and $F_{4}$ in Mond's classification ([19]) also have less than or equal to 5 generators in the set of liftable vector fields, since they all admit one-parameter stable unfoldings $\mathcal{A}$-equivalent to $F$.

6.9. Lift $(f)$ for $f(x, y)=\left\{\left(x, y^{3}+x y\right),\left(x, y^{2}\right)\right\}$.

Let $f=\left\{f_{1}, f_{2}\right\}$ be the plane to plane bigerm defined by $f_{1}(x, y)=\left(x, y^{3}+x y\right)$ and $f_{2}(x, y)=\left(x, y^{2}\right)$. Consider the one-parameter stable unfolding $F=\left\{F_{1}, F_{2}\right\}$ defined by

$$
\left\{\begin{array}{l}
\left(x, y^{3}+x y, z\right) \\
\left(x, y^{2}+z, z\right)
\end{array} .\right.
$$

It is not hard to see that $\operatorname{Lift}\left(F_{1}\right)=\left\langle 2 X \frac{\partial}{\partial X}+3 Y \frac{\partial}{\partial Y}, 9 Y \frac{\partial}{\partial X}-2 X^{2} \frac{\partial}{\partial Y}, \frac{\partial}{\partial Z}\right\rangle_{C_{0}}$ and $\operatorname{Lift}\left(F_{2}\right)=\left\langle\frac{\partial}{\partial X}, Y \frac{\partial}{\partial Y}+Z \frac{\partial}{\partial Z}, \frac{\partial}{\partial Y}+\frac{\partial}{\partial Z}\right\rangle_{C_{0}}$ where $(X, Y, Z)$ are the variables in the target. So $\operatorname{Lift}(F)=\operatorname{Lift}\left(F_{1}\right) \cap \operatorname{Lift}\left(F_{2}\right)=$

$$
\left\langle(Z-Y) \frac{\partial}{\partial Z}, 2 X \frac{\partial}{\partial X}+3 Y \frac{\partial}{\partial Y}+3 Z \frac{\partial}{\partial Z}, 9 Y \frac{\partial}{\partial X}-2 X^{2} \frac{\partial}{\partial Y}-2 X^{2} \frac{\partial}{\partial Z}\right\rangle_{C_{0}} .
$$

To apply Theorem 2, we consider $g(x, y, z)=\left(x, y, z^{2}\right)$. Then $\operatorname{Lift}(g)=\left\langle\frac{\partial}{\partial X}, \frac{\partial}{\partial Y}, Z \frac{\partial}{\partial Z}\right\rangle_{C_{0}}$. So $\operatorname{Lift}(F) \cap \operatorname{Lift}(g)=$

$$
\begin{gathered}
\left\langle 2 X \frac{\partial}{\partial X}+3 Y \frac{\partial}{\partial Y}+3 Z \frac{\partial}{\partial Z}, 9 Y^{2} \frac{\partial}{\partial X}-2 X^{2} Y \frac{\partial}{\partial Y}-2 X^{2} Z \frac{\partial}{\partial Z},\right. \\
\left.\left(27 Y Z+4 X^{3}\right) \frac{\partial}{\partial X}+\left(-6 X^{2} Z+6 X^{2} Y\right) \frac{\partial}{\partial Y},\left(Z^{2}-Y Z\right) \frac{\partial}{\partial Z}\right\rangle_{C_{0}} .
\end{gathered}
$$

And finally $\operatorname{Lift}(f)=\left\langle 2 X \frac{\partial}{\partial X}+3 Y \frac{\partial}{\partial Y}, 9 Y^{2} \frac{\partial}{\partial X}-2 X^{2} Y \frac{\partial}{\partial Y}\right\rangle_{C_{0}}$.

Remark 1. Few complete classifications of simple multigerms are known, namely [12], 32, 8] and [23]. Based on these classifications, it seems that most simple germs, except for a few cases which can be excluded by the multiplicity (21]), admit one-parameter stable unfoldings. This suggests that the method followed above can be applied to most simple germs.

\section{The CASE $n>p$}

Let $\bar{f}:\left(\mathbb{K}^{n}, 0\right) \rightarrow\left(\mathbb{K}^{p}, 0\right)$ be a corank 1 simple germ with $n>p$. By [25], $\bar{f}$ is $\mathcal{A}$-equivalent to

$$
f\left(x_{1}, \ldots, x_{p}, \ldots, x_{n}\right)=\left(x_{1}, \ldots, x_{p-1}, g\left(x_{1}, \ldots, x_{p}\right)+\sum_{j=p+1}^{n} a_{j} x_{j}^{2}\right),
$$


where $a_{j}= \pm 1,(p+1 \leq j \leq n)$. Let $f_{0}:\left(\mathbb{K}^{p}, 0\right) \rightarrow\left(\mathbb{K}^{p}, 0\right)$ be the germ such that $f_{0}\left(x_{1}, \ldots, x_{p}\right)=\left(x_{1}, \ldots, x_{p-1}, g\left(x_{1}, \ldots, x_{p}\right)\right)$.

Proposition 8. $\operatorname{Lift}(f)=\operatorname{Lift}\left(f_{0}\right)$.

\section{Proof of Proposition 8}

First suppose $\eta \in \operatorname{Lift}(f)$, by definition there exists $\xi \in \theta_{0}(n)$ such that $\eta \circ$ $f\left(x_{1}, \ldots, x_{n}\right)=d f \circ \xi\left(x_{1}, \ldots, x_{n}\right)$. In particular, $\eta \circ f\left(x_{1}, \ldots, x_{p}, 0, \ldots, 0\right)=d f \circ$ $\xi\left(x_{1}, \ldots, x_{p}, 0, \ldots, 0\right)$ and therefore $\eta \circ f_{0}\left(x_{1}, \ldots, x_{p}\right)=d f_{0} \circ \bar{\xi}\left(x_{1}, \ldots, x_{p}\right)$ where

$$
\bar{\xi}\left(x_{1}, \ldots, x_{p}\right)=\left(\begin{array}{c}
\xi_{1}\left(x_{1}, \ldots, x_{p}, 0, \ldots, 0\right) \\
\vdots \\
\xi_{p}\left(x_{1}, \ldots, x_{p}, 0, \ldots, 0\right)
\end{array}\right) .
$$

Therefore, $\eta \in \operatorname{Lift}\left(f_{0}\right)$ and so $\operatorname{Lift}(f) \subset \operatorname{Lift}\left(f_{0}\right)$.

Now suppose $\eta_{0} \in \operatorname{Lift}\left(f_{0}\right)$, by definition there exists $\xi_{0} \in \theta_{0}(p)$ such that $\eta_{0} \circ f_{0}=d f_{0} \circ \xi_{0}$. Note that $d f_{0} \circ \xi_{0}=d f \circ \xi$ where

$$
\xi\left(x_{1}, \ldots, x_{n}\right)=\left(\begin{array}{c}
\xi_{0_{1}}\left(x_{1}, \ldots, x_{p}\right) \\
\vdots \\
\xi_{0_{p}}\left(x_{1}, \ldots, x_{p}\right) \\
0 \\
\vdots \\
0
\end{array}\right) .
$$

We have that $\eta_{0} \circ f\left(x_{1}, \ldots, x_{n}\right)=$

$$
\left(\begin{array}{c}
\eta_{0_{1}} \circ f\left(x_{1}, \ldots, x_{p}, 0, \ldots, 0\right) \\
\vdots \\
\eta_{0_{p}} \circ f\left(x_{1}, \ldots, x_{p}, 0, \ldots, 0\right)
\end{array}\right)+\left(\begin{array}{c}
\eta_{0_{1}} \circ f\left(x_{1}, \ldots, x_{n}\right)-\eta_{0_{1}} \circ f\left(x_{1}, \ldots, x_{p}, 0, \ldots, 0\right) \\
\vdots \\
\eta_{0_{p}} \circ f\left(x_{1}, \ldots, x_{n}\right)-\eta_{0_{p}} \circ f\left(x_{1}, \ldots, x_{p}, 0, \ldots, 0\right)
\end{array}\right)
$$

The first matrix is equal to $\eta_{0} \circ f_{0}=d f_{0} \circ \xi_{0}=d f \circ \xi$ and, by Hadamard's Lemma ([7), there exist functions $\bar{\xi}_{p+1}, \ldots, \bar{\xi}_{n}$ such that the second matrix is equal to

$$
d f \circ\left(\begin{array}{c}
\eta_{0_{1}} \circ f\left(x_{1}, \ldots, x_{n}\right)-\eta_{0_{1}} \circ f\left(x_{1}, \ldots, x_{p}, 0, \ldots, 0\right) \\
\vdots \\
\eta_{0_{p}} \circ f\left(x_{1}, \ldots, x_{n}\right)-\eta_{0_{p}} \circ f\left(x_{1}, \ldots, x_{p}, 0, \ldots, 0\right) \\
0 \\
\bar{\xi}_{p+1} \\
\vdots \\
\overline{\bar{\xi}}_{n}
\end{array}\right) .
$$

So $\eta_{0} \in \operatorname{Lift}(f)$ and the proposition is proved.

The proposition holds for multigerms too since the above proof can be repeated for each branch. Thus, we can obtain the following

Example 7.1. Let $f=\left\{f_{1}, f_{2}\right\}:\left(\mathbb{K}^{n},\{0,0\}\right) \rightarrow\left(\mathbb{K}^{2}, 0\right), n>2$, be the bigerm defined by

$$
\left\{\begin{array}{l}
f_{1}\left(x, y, u_{1}, \ldots, u_{n-2}\right)=\left(x, y^{3}+x y+\sum_{i=1}^{n-2} a_{i} u_{i}^{2}\right) \\
f_{2}\left(x, y, u_{1}, \ldots, u_{n-2}\right)=\left(x, y^{2}+\sum_{i=1}^{n-2} b_{i} u_{i}^{2}\right)
\end{array}\right.
$$


where $a_{i}= \pm 1$ and $b_{i}= \pm 1,(1 \leq i \leq n-2)$. Then Lift $(f)=\left\langle 2 X \frac{\partial}{\partial X}+\right.$ $\left.3 Y \frac{\partial}{\partial Y}, 9 Y^{2} \frac{\partial}{\partial X}+2 X^{2} Y \frac{\partial}{\partial Y}\right\rangle_{C_{0}}$.

\section{ACKNOWLEDGEMENTS}

The authors would like to thank Mohammed Salim Jbara Al-Bahadeli for his pointing out careless mistakes in calculations for concrete liftable vector fields

\section{REFERENCES}

[1] V. I. Arnol'd, Wave front evolution and equivariant Morse lemma, Commun Pure Appl. Math. 29 (1976) 557-582.

[2] V. I. Arnol'd, S. M. Gusein-Zade and A. N. Varchenko, Singularities of Differentiable Maps I (Monographs in Mathematics 82) (Birkhäuser, Boston, 1985).

[3] J. W. Bruce and T. J. Gaffney, Simple singularities of mappings $\mathbb{C}, 0 \rightarrow \mathbb{C}^{2}, 0$, J. London Math. Soc. 26 (1982) 465-474.

[4] J. W. Bruce and J. M. West, Functions on a crosscap, Math. Proc. Cambridge Philos. Soc. 123 (1998) 19-39.

[5] T. Cooper, D. Mond and R. Wik Atique, Vanishing topology of codimension 1 multi-germs over $\mathbb{R}$ and $\mathbb{C}$, Compositio Math. 131 (2002) 121-160.

[6] J. Damon, $\mathcal{A}$-equivalence and the equivalence of sections of images and discriminants, in Singularity Theory and its Applications, Part I (Coventry, 1988/1989) 93-121 Lecture Notes in Math. 1462 Springer, Berlin, 1991.

[7] C. G. Gibson, Singular points of smooth mappings. Research Notes in Mathematics, 25. Pitman (Advanced Publishing Program), Boston, Mass.-London, 1979. iv+239 pp.

[8] C. A. Hobbs and N. Kirk, On the classification and bifurcation of multigerms of maps from surfaces to 3-space, Math. Scand. 89 (2001), no. 1, 5796.

[9] M. P. Holland and D. Mond, Stable mappings and logarithmic relative symplectic forms, Math. Z. 231 (1999) 605-623.

[10] K. Houston, Augmentation of singularities of smooth mappings, Internat. J. Math. 15 (2004), $111-124$.

[11] K. Houston and D. Littlestone, Vector fields liftable over corank 1 stable maps, arXiv.org math (2009) no. 0905.0556.

[12] P. A. Kolgushkin and R. R. Sadykov, Simple singularities of multigerms of curves, Rev. Mat. Complut. 14 (2001) 311-344.

[13] E. J. N. Looijenga, Isolated Singular Points on Complete Intersections, London Mathematical Society Lecture Note Series, 77, Cambridge University Press, Cambridge, 1984.

[14] J. Mather, Stability of $C^{\infty}$ mappings, III. Finitely determined map-germs, Publ. Math. Inst. Hautes Études Sci. 35 (1969) 127-156.

[15] J. Mather, Stability of $C^{\infty}$ mappings, IV, Classification of stable map-germs by $\mathbb{R}$-algebras, Publ. Math. Inst. Hautes Études Sci. 37 (1970) 223-248.

[16] J. Mather, Stability of $C^{\infty}$-mappings V. Transversality, Adv. in Math. 4 (1970) 301-336.

[17] J. Mather, Stability of $C^{\infty}$-mappings VI. The nice dimensions, Lecture Notes in Math. 192 (1971) 207-253.

[18] Y. Mizota and T. Nishimura, Multicusps, in Real and Complex Singularities, Contemporary Mathematics, vol. 569, Amer. Math. Soc., Providence RI, 2012, pp. 115-121.

[19] D. Mond, On the classification of germs of maps from $\mathbb{R}^{2}$ to $\mathbb{R}^{3}$, Proc. London Math. Soc. 50 (1985), 333-369.

[20] B. Morin, Formes canoniques des singularites d'une application differentiable, Comptes Rendus 260(1965) 5662-5665 and 6503-6506.

[21] T. Nishimura, $\mathcal{A}$-simple multigerms and $\mathcal{L}$-simple multigerms, Yokohama Math. J. 55 (2010) 93-104.

[22] R. Oset Sinha, M. A. S. Ruas and R. Wik Atique, Classifying codimension two multigerms, Math. Z. DOI 10.1007/s00209-014-1326-2.

[23] R. Oset Sinha, M. A. S. Ruas and R. Wik Atique, On the simplicity of multigerms, To appear in Math. Scand. 
[24] J. H. Rieger, Families of maps from the plane to the plane, J. London Math. Soc. 36 (1987) $351-369$.

[25] J. H. Rieger and M. A. S. Ruas, Classification of $\mathcal{A}$-simple germs from $\mathbb{K}^{n}$ to $\mathbb{K}^{2}$. Compositio Math. 79 (1991), no. 1, 99108.

[26] J. H. Rieger, M. A. S. Ruas and R. Wik Atique, M-deformations of $\mathcal{A}$-simple germs from $\mathbb{R}^{n}$ to $\mathbb{R}^{n+1}$, Math. Proc. Camb. Phil. Soc. 144 (2008) 181-195.

[27] K. Saito, Theory of logarithmic differential forms and logarithmic vector fields, J. Fac. Sci. Univ. Tokyo Sect. IA Math. 27 (1980) 265-291.

[28] C. T. C. Wall, Finite determinacy of smooth map-germs, Bull. London Math. Soc. 13 (1981) 481-539.

[29] H. Whitney, The general type of singularities of a set of $2 n-1$ smooth functions of $n$ variables, Duke Math. J. 10 (1943) 161-172.

[30] H. Whitney, The singularities of a smooth $n$-manifold in $(2 n-1)$-space, Ann. of Math. 45 (1944) 247-293.

[31] H. Whitney, On singularities of mappings of Euclidean spaces. Mappings of the plane to the plane, Ann. of Math. 62 (1955) 374-410.

[32] R. Wik Atique On the classification of multi-germs of maps from $\mathbb{C}^{2}$ to $\mathbb{C}^{3}$ under $\mathcal{A}$ equivalence, in J.W.Bruce and F.Tari(eds.) Real and Complex Singularities, Research Notes in Maths Series, Chapman \& Hall / CRC (2000), 119-133.

Research Group of Mathematical Sciences, Research Institute of Environment and Information Sciences, Yokohama National University, Yokohama 240-8501, JAPAN.

E-mail address: nishimura-takashi-yx@ynu.jp

Department of Mathematics, Federal University of São Carlos, São Carlos, SP, BRAZIL

E-mail address: Raul.Oset@uv.es

ICMC, University of SÃo Paulo, São Carlos, SP, Brazil

E-mail address: maasruas@icmc.usp.br

iCMC, University of São Paulo, São Carlos, SP, Brazil

E-mail address: rwik@icmc.usp.br 